Supplementary Information

\title{
Persistent Multiexcitons from Polymers with Pendent Pentacenes
}

Lauren M. Yablon, ${ }^{1, \ddagger}$ Samuel N. Sanders, ${ }^{1,+}$ Hua Li, ${ }^{2}$ Kaia R. Parenti, ${ }^{1}$ Elango Kumarasamy, ${ }^{1}$ Kealan J. Fallon, ${ }^{1}$ Michael J. A. Hore,${ }^{3}$ Angelo Cacciuto, ${ }^{1}$ Matthew Y. Sfeir, ${ }^{4,5 *}$ and Luis M. $\mathrm{Campos}^{2 *}$

${ }^{1}$ Department of Chemistry, Columbia University, New York, New York 10027, United States ${ }^{2}$ College of Chemistry, Chemical Engineering and Materials Science, Collaborative Innovation Center of Suzhou Nano Science and Technology, Soochow University, Suzhou 215123, P. R. China.

${ }^{3}$ Department of Macromolecular Science and Engineering, Case Western Reserve University, 10900 Euclid Avenue, Cleveland, OH 44106, United States

${ }^{4}$ Photonics Initiative, Advanced Science Research Center, City University of New York, New York, New York 10031, United States

${ }^{5}$ Department of Physics, Graduate Center, City University of New York, New York, New York 10016, United States

these authors contributed equally

Correspondence to: msfeir@gc.cuny.edu, lcampos@columbia.edu

Table of Contents:

I. Transient Absorption Spectroscopy and Photoluminescence Spectroscopy....................2

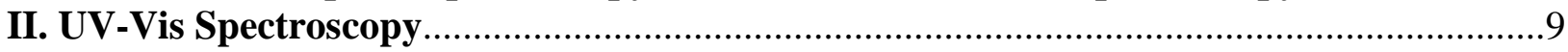

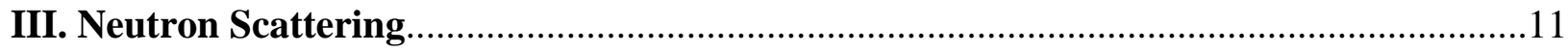

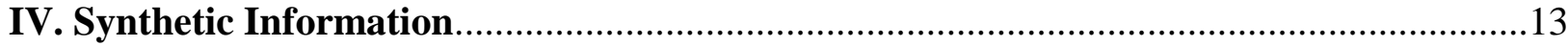

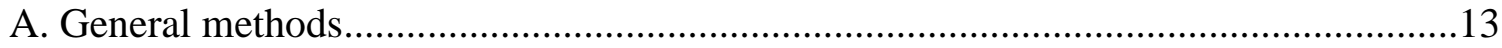

B. General protocol for the synthesis of pentacene polymers.....................................14

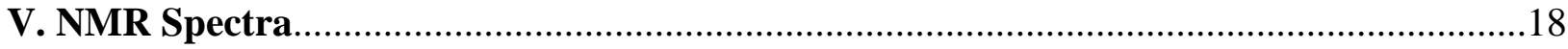

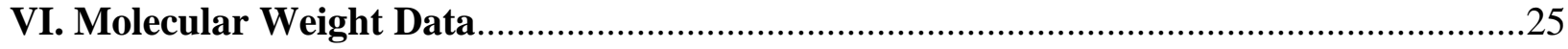

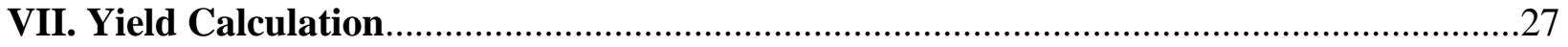

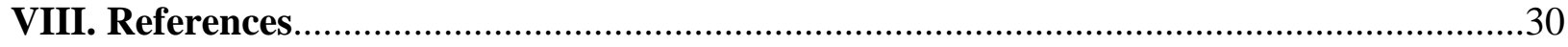




\section{Transient Absorption and Photoluminescence Spectroscopy}

\section{Instrumentation}

A detailed description about the transient absorption (TA) spectroscopy employed for these studies has been previously reported. ${ }^{1}$ A resonant pump pulse of $\sim 100$ fs was generated with a $1 \mathrm{KHz}$ amplified Ti:Sapphire system with an optical parametric amplifier. A femtosecond supercontinuum probe in a thin sapphire plate is also generated from the laser. The delay in the probe is controlled mechanically. A fiber laser (Leukos) is used to generate a nanosecond supercontiunuum probe pulse. Longer delay times are measured using an electronically delayed configuration. In the two probe measurements, the pump pulse is the same. All sample solution concentrations were below $520 \mu \mathrm{M}$.

An upconversion technique was used to measure ultrafast photoluminescence data. A $600 \mathrm{~nm}, 100 \mathrm{fs}$ laser pulse was used to excite the sample in toluene. A "gate" pulse in a nonlinear crystal was used to mix the spontaneous emission to achieve a sum frequency. The instantaneous photoluminescence intensity was proportional to the unconverted optical signal magnitude. The unconverted optical signal was measured as intervals of delay between excitation and gate pulse. The measurement had $\sim 250 \mathrm{fs}$ time resolution, and a spectral resolution of $\sim 10 \mathrm{~nm}$.

Figure S1 shows the TA sensitization spectrum of PePNo24. The pentacene polymer was dissolved in a $15 \mathrm{mM}$ solution of anthracene in toluene. The solution was excited at $360 \mathrm{~nm}$, which selectively excites the anthracene and not the pentacene units on the polymers. The anthracene molecules then undergo intersystem crossing to produce single triplets. The triplets are then transferred to pentacene through collisions. We are then able to observe what a free triplet absorption spectrum on the pentacene polymer looks like. 


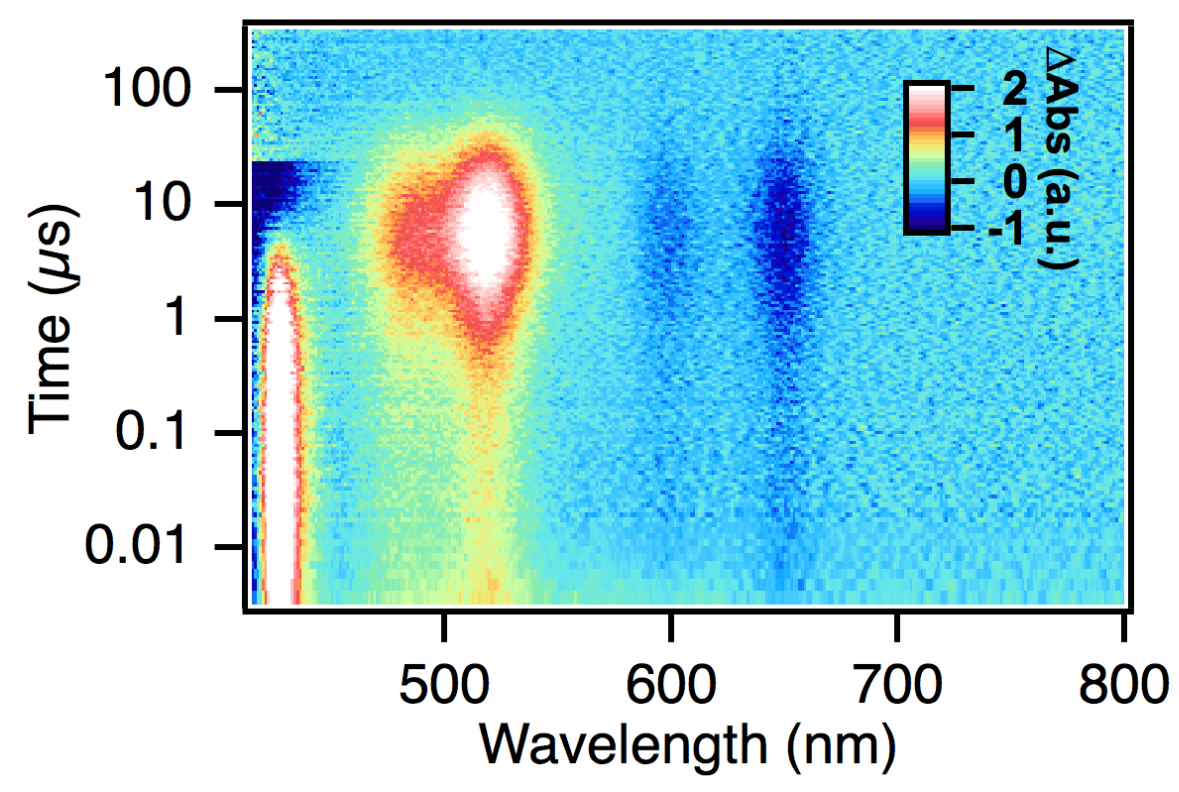

Figure S1: Sensitization of PePNo24 in toluene $(\sim 100 \mu \mathrm{M})$ using anthracene $(15 \mathrm{mM})$. The solution was excited at $360 \mathrm{~nm}(\sim 400 \mu \mathrm{W})$ to selectively excite anthracene.

The data was analyzed using Global Analysis to find the time constants for the data. Figure S2 shows the raw data and Global Analysis fits taken at $450 \mathrm{~nm}$ and $520 \mathrm{~nm}$. Four exponents were needed to fit the data adequately.

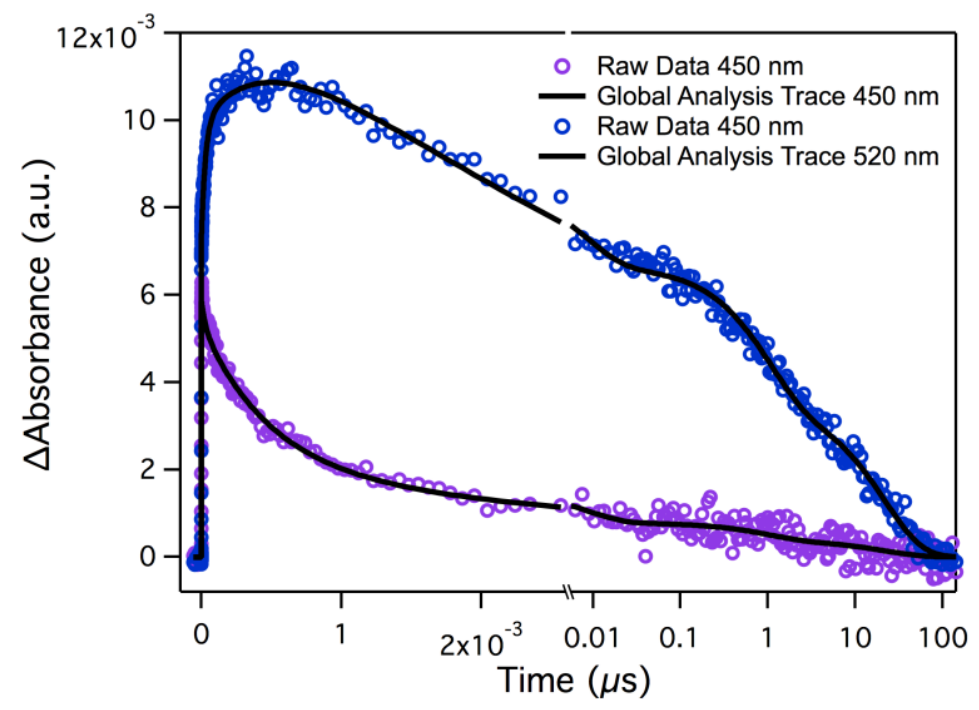

Figure S2: Kinetic fits of PePNo24 generated by Global Analysis. The trace at $450 \mathrm{~nm}$ shows the decay of singlet, and the trace at $520 \mathrm{~nm}$ shows the rise and decay of the triplet. 
The time constants for singlet fission in the five polymers explored in this study are shown in Table S1. $\tau_{\mathrm{S}}$ is the time constant for the singlet state. $\tau_{\mathrm{I}}$ is the time constant for the intermediate state that is both singlets and triplets. $\tau_{2 \mathrm{~T}}$ is the time constant for the two mobile triplets, and $\tau_{\mathrm{T}}$ is the time constant for the single triplet left after geminate recombination.

Table S1: Pentacene polymer decay time constants determined by Global Analysis.

\begin{tabular}{|c|c|c|c|c|}
\hline & $\tau_{\mathrm{S}}(\mathrm{ps})$ & $\tau_{\mathrm{I}}(\mathrm{ps})$ & $\tau_{2 \mathrm{~T}}(\mathrm{~ns})$ & $\tau_{\mathrm{T}}(\mu \mathrm{s})$ \\
\hline PePNo8 & 22.0 & 711 & 415 & 23 \\
\hline PePNo10 & 26.0 & 789 & 471 & 21 \\
\hline PePNo16 & 27.1 & 678 & 839 & 28 \\
\hline PePNo24 & 28.6 & 486 & 1000 & 22 \\
\hline PePNo33 & 21 & 420 & 862 & 15 \\
\hline
\end{tabular}

Figure S3 shows a comparison of the raw data set and the modeled data set resulting from a global analysis using the time constants in Table S1. In applying a model to the data, we chose the simplest picture that communicates the most essential features. We believe that our discrete model captures the essential physical representation of the polymer, in which a small number of relatively stable conformations dominate the average behavior of the ensemble and give rise to a combination of well-ordered and disordered regions. We favored this approach over more complex distributed kinetic models (e.g., stretched exponentials that represent power law distributions with no characteristic time constant) due to the small number of time constants needed to give an accurate representation of the data. While there is likely some fluctuation around the average time constants reported above, these fluctuations have minimal effect on the fitting and interpretation of the data.

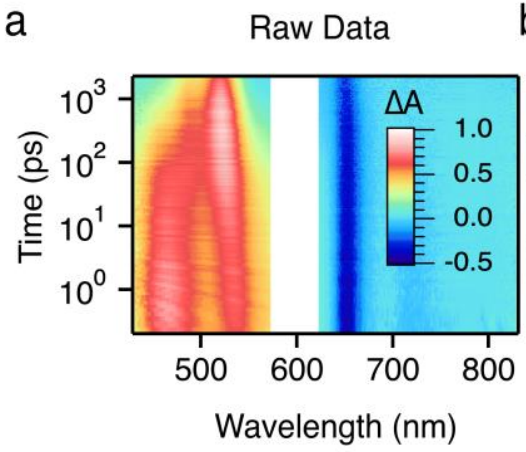

Wavelength $(\mathrm{nm})$ b Data Fit

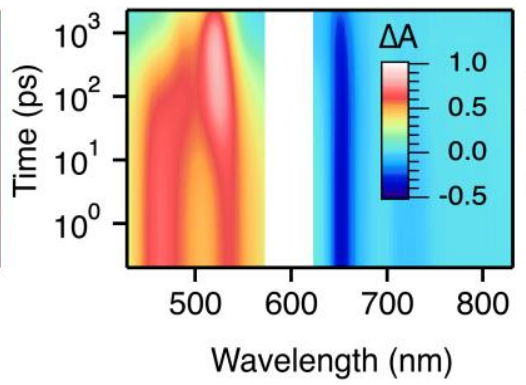

C

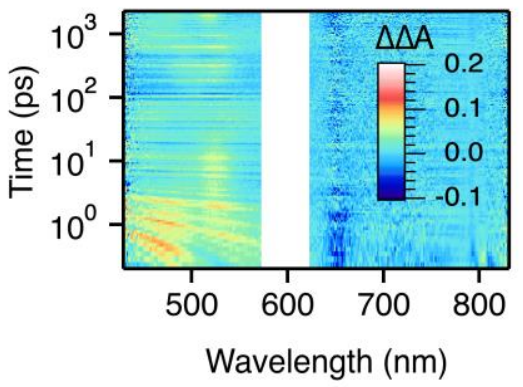


Figure S3: Comparison of the (a) raw transient absorption data for PePNo24, (b) modeled data set using the fit parameters from a global analysis of the raw data using a three species first order kinetic fit, (c) residual matrix showing the difference between the raw data set and the modeled data set.

Figures S4 shows the kinetic traces from the transient absorption spectra of two different concentrations of PePNo24, $520 \mu \mathrm{M}$ and $90 \mu \mathrm{M}$. The kinetic traces at $450 \mathrm{~nm}$ (left) show the decay of the singlet feature at the two different concentrations. Kinetic cuts were also taken at $520 \mathrm{~nm}$ where there is a large absorption of the triplet and little from the singlet. From these kinetic traces, it became apparent that the decay times for singlets and triplets in different concentrations of PePNo24 remain constant. A constant decay time over different concentrations implies that singlet fission is occurring on pentacenes in a single polymer chain. Additionally, Richard Friend and co-workers demonstrated that bis(triisopropylsilylethynyl (TIPS)) pentacene in solution does not undergo singlet fission until it reaches a concentration of $7.9 \times 10^{-3} \mathrm{~mol} / \mathrm{L}$ or $7900 \mu \mathrm{M},{ }^{3}$ which is an order of magnitude more concentrated than the solutions used in this study.
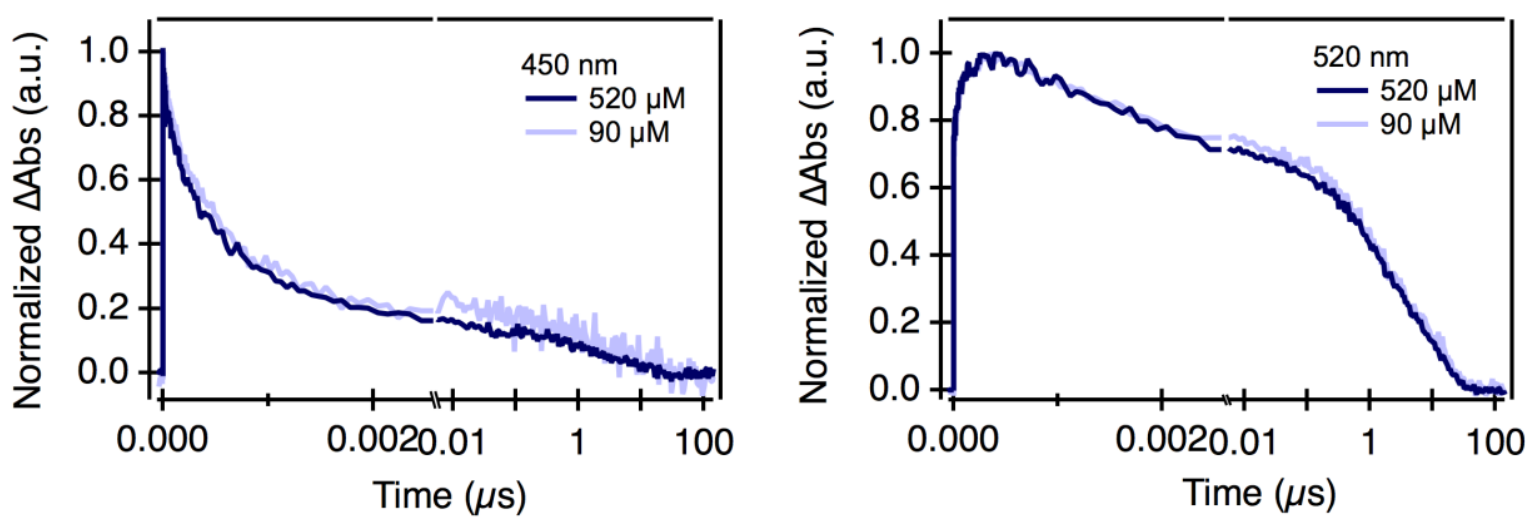

Figure S4: Kinetic traces of different concentrations of PePNo24 excited at $600 \mathrm{~nm}(250 \mu \mathrm{W})$. Left shows the singlet decay at $450 \mathrm{~nm}$ and right shows the triplet rise and decay at $520 \mathrm{~nm}$.

Figure S5 shows kinetic traces at $520 \mathrm{~nm}$ from the TA of PePNo24 at different laser powers. The signal decay remained the same as the laser power increased. Since there was no dependence on the power in this range, we concluded that multiple excitons interactions on an excited polymer chain are not occurring. We ensured that the laser power for the data taken fell 
within the range of $375 \mu \mathrm{J} / \mathrm{cm}^{2}$ and $25 \mu \mathrm{J} / \mathrm{cm}^{2}$, as this is where laser power did not affect the time constants observed. It is unclear if one exciton bleaches one or more chromphore along the polymer. Without extended bleaching of the polymer, the highest fluence value would correspond to $\sim 1$ singlet/11 monomers on average (using Poisson statistics and a monomer molar absorptivity of $\sim 21,000 \mathrm{M}^{-1} \mathrm{~cm}^{-1}$ ). This value is a factor of 2 higher than the molecular weight value at which the triplet-triplet annihilation rate constant saturates $(\sim 20$ repeat units or $\mathrm{Mn}=17 \mathrm{~kg} / \mathrm{mol}$ ). This is suggestive (though not conclusive) that some extended bleaching of the chains occurs that suppresses singlet annihilation.

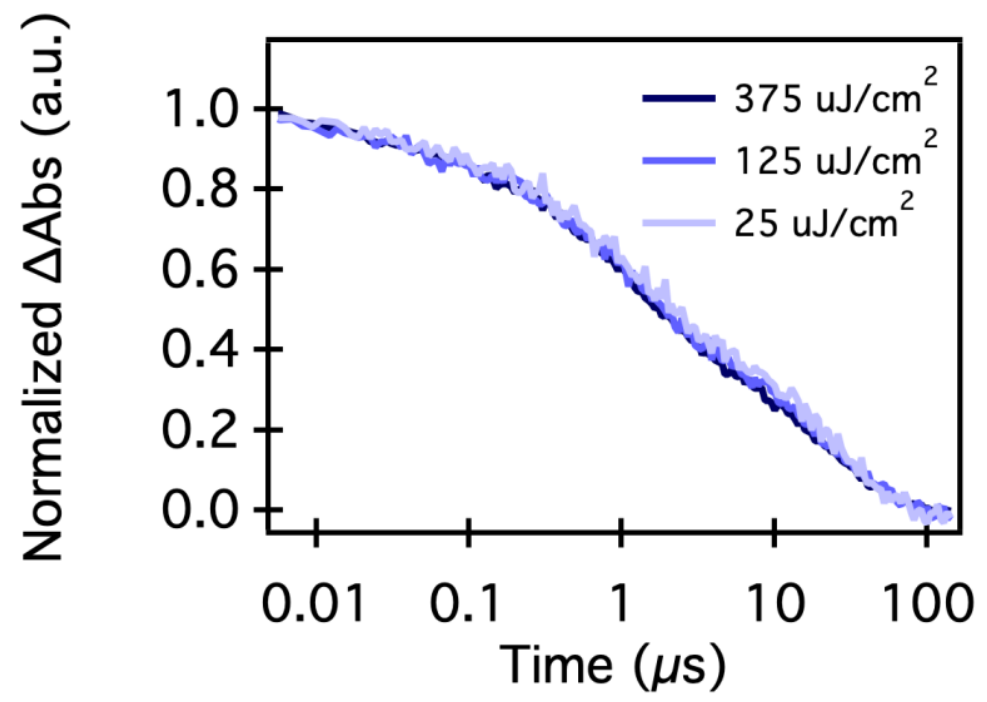

Figure S5: Kinetic traces at $520 \mathrm{~nm}$ of PePNo24 at varying laser powers excited at $600 \mathrm{~nm}$.

Transient absorption data was taken of PePNo24 in the near IR, Figure S6. Previous studies have suggested that triplet pairs in close proximity will produce a characteristic triplet absorption feature in the infrared. ${ }^{1,5}$ Our NIR data support do not show a characteristic photoinduced absorption indicative of a strongly bound triplet pair. ${ }^{4}$ Global Analysis confirmed that the only NIR signal we observed results from photoexcited singlets, similar to monomeric TIPS pentacene. By comparing the visible and NIR deconvoluted TA spectra we see that the different time constants are consistent with what have assigned to disordered and aligned singlet species. 
a
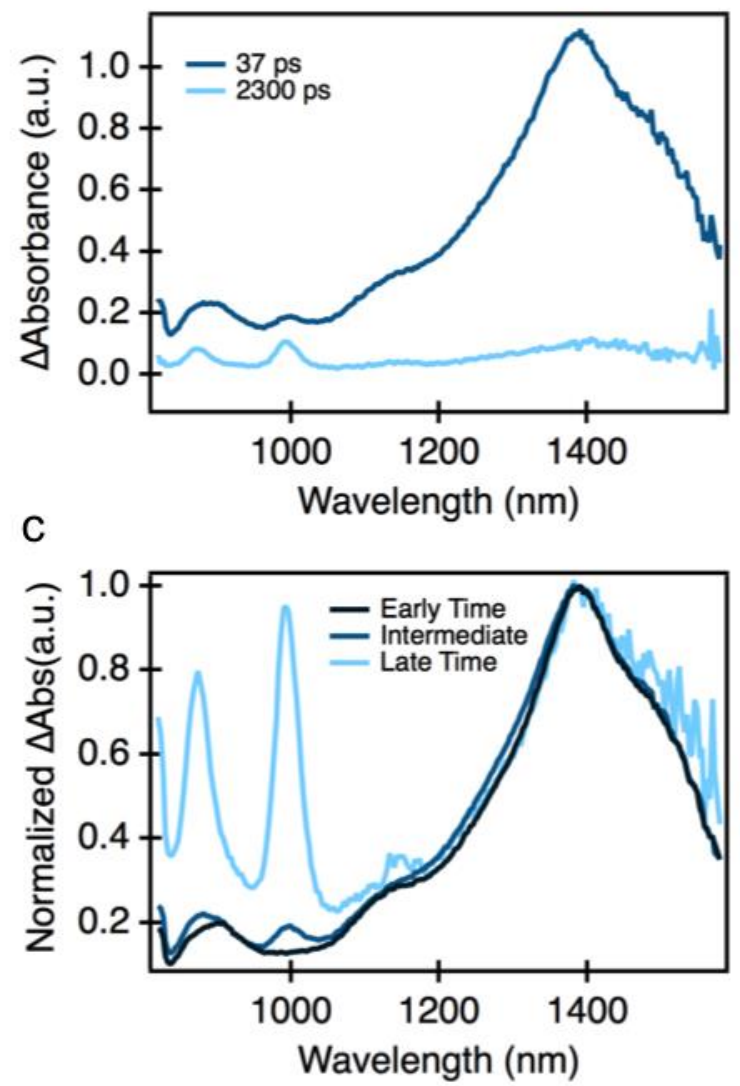

b

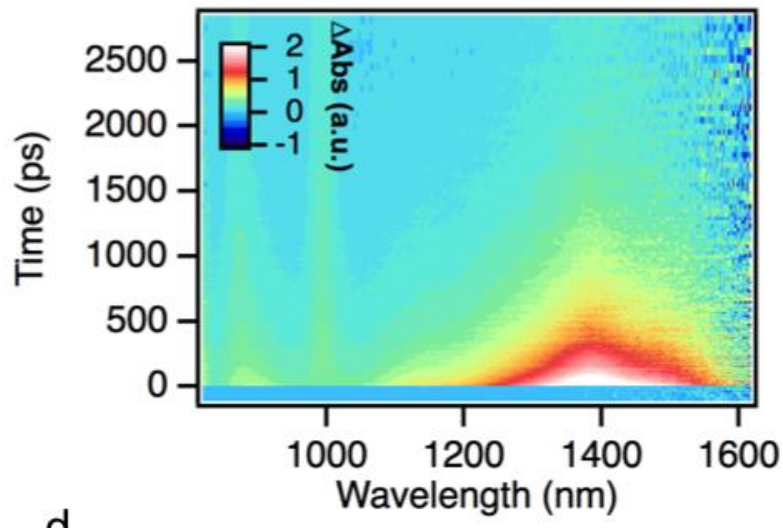

d

\begin{tabular}{|c|c|}
\hline & Ts (ps) \\
\hline Early Time & 27 \\
\hline Intermediate & 454 \\
\hline Late Time & $8000^{*}$ \\
\hline
\end{tabular}

Figure S6: (a) Spectral cuts taken from the NIR TA spectrum of PePNo24 at early and late times. (b) TA spectrum of PePNo24 in the NIR region excited at 600nm (300 $\mu \mathrm{W})$. (c) Normalized singlet spectra from Global Analysis. (d) Time constants of the different singlet decays. *This number is not accurate as it is well beyond the lifetime of the measurement and requires Global Analysis to extrapolate.

Transient absorption spectra of polymer films were taken in different polymer matrix (Figure S7). These included a neat film, a polymethylmethacrylate (PMMA) matrix, and a polystyrene matrix. Singlet fission is observed in all three of these films when the films were excited at $600 \mathrm{~nm}$ with the same power. They all undergo fast singlet fission to yield triplets. 


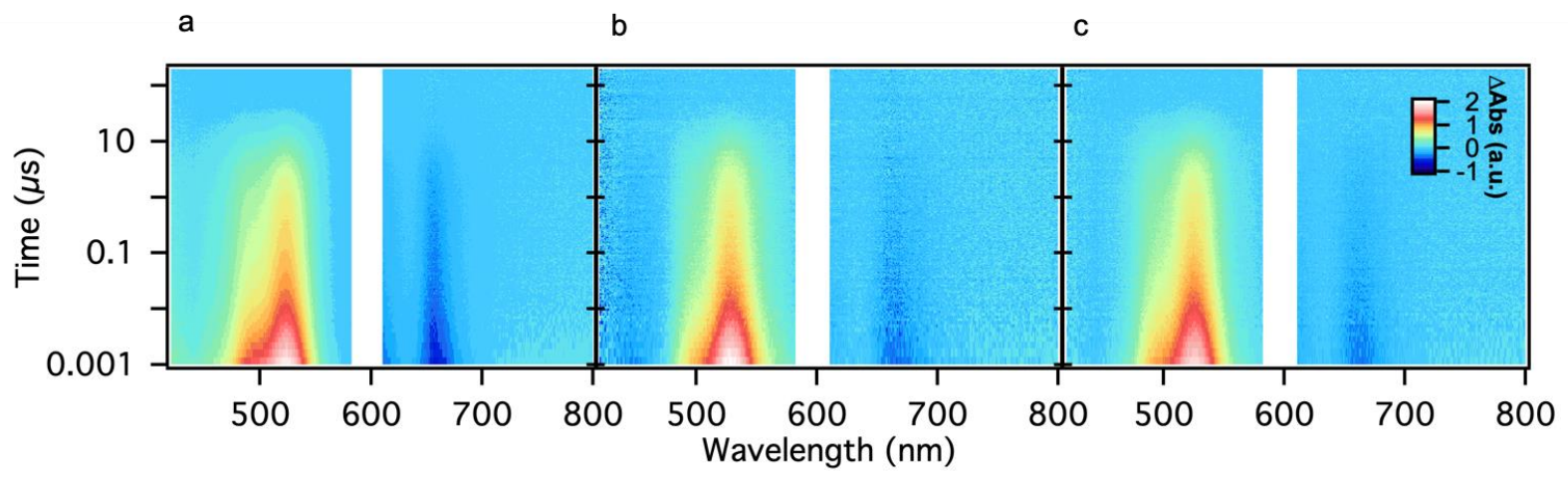

Figure S7: Transient absorption spectra of polymer films. The films were excited at $600 \mathrm{~nm}$ $(250 \mu \mathrm{W})$. (a) TA spectrum of neat polymer film. (b) TA spectrum of polymer in a PMMA matrix. (c) TA spectrum of polymer in polystyrene matrix.

Figure S8 shows the kinetic traces of the transient absorption spectroscopy at $530 \mathrm{~nm}$. These traces were compared for the three different films discussed above. They exhibited similar kinetics, and therefore the triplets in these films have similar lifetimes. This demonstrates that the medium the polymer is in does not drastically affect triplet lifetime.

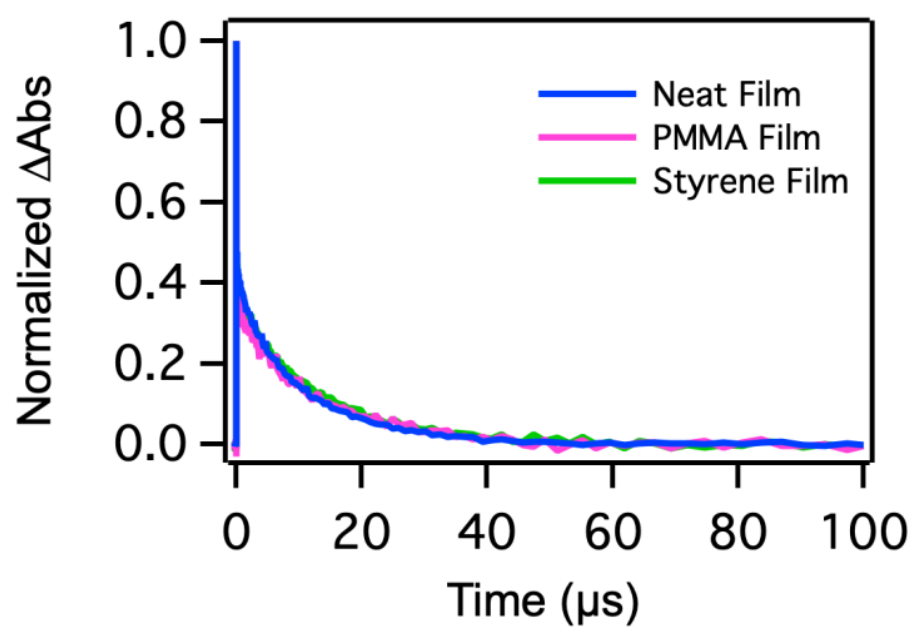

Figure S8: Kinetic traces of PePNo29 films in different matrices. The films were excited at 600 $\mathrm{nm}(250 \mu \mathrm{W})$. 


\section{UV-Vis Spectroscopy}

\section{Instrumentation}

UV/Vis spectra were taken on an Agilent Cary 60 UV-Vis Spectrophotometer.

UV/Vis spectroscopy was taken of the pentacene monomer and PePNo24 in toluene (Figure S9). The spectra are nearly identical, and exhibit the characteristic pentacene peaks around $600 \mathrm{~nm}$. Since the monomer and polymer spectra are the same, we concluded that the polymer is not aggregating. To further confirm this, a solution of the polymer in a mixture of toluene and acetonitrile, a bad solvent, was prepared to intentionally induce aggregation in order to obtain a spectrum of an aggregated sample. Upon aggregation, the polymer absorption onset red shifted, and the peak at $325 \mathrm{~nm}$ broadened. ${ }^{3}$ Since these phenomena are not observed in the toluene solution we conclude that the polymer is well dissolved while we carry out spectroscopic studies.

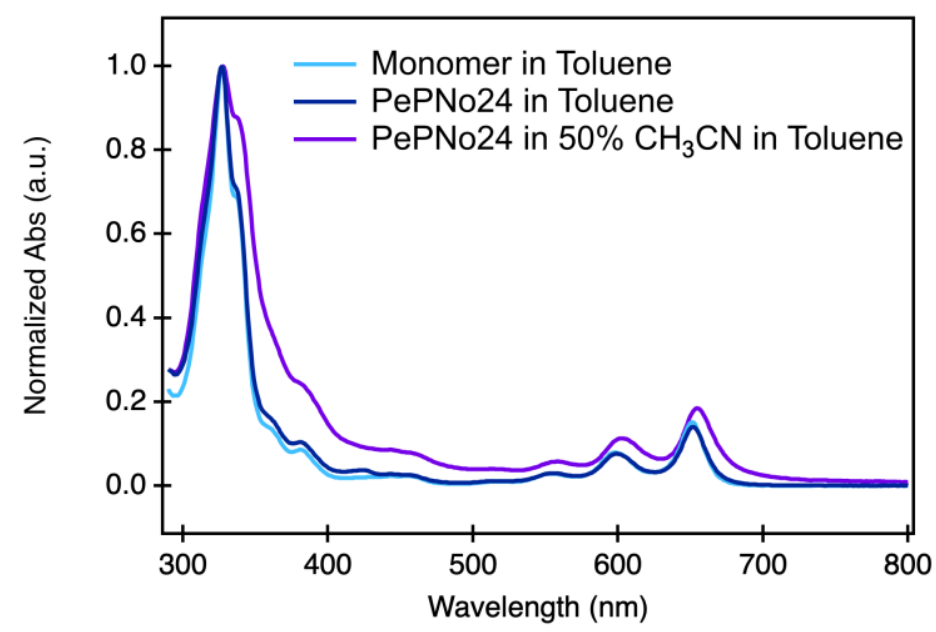

Figure S9: UV/Vis spectrum of monomer in toluene and UV/Vis spectra of PePNo24 in toluene and PePNo24 in 50\% acetonitrile in toluene.

The UV/Vis absorptions of PePNo24 and its corresponding monomer are red shifted compared to the absorption of TIPS pentacene (Figure S10). Because of this, the emission spectrum of PePNo24 is red shifted in comparison to TIPS pentacene. 


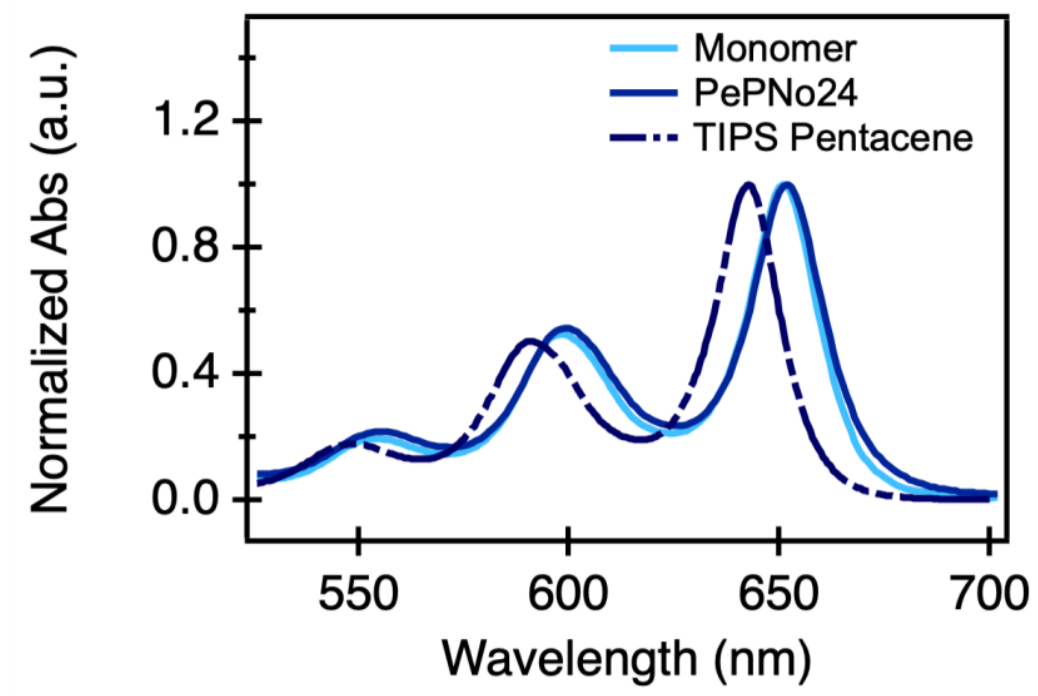

Figure S10: UV/Vis spectrum of monomer, PePNo24, and TIPS pentacene in toluene.

Figure S11 shows the UV/Vis spectra of the polymer films and the polymer in solution. The film spectra are slightly broadened and red-shifted, but the three vibrionic peaks are still prevalent. The lack of a significant change in peak shape when the pentacene units are highly coupled in the film, demonstrates that peak shape cannot be used to determine the level of neighboring pentacene interactions.

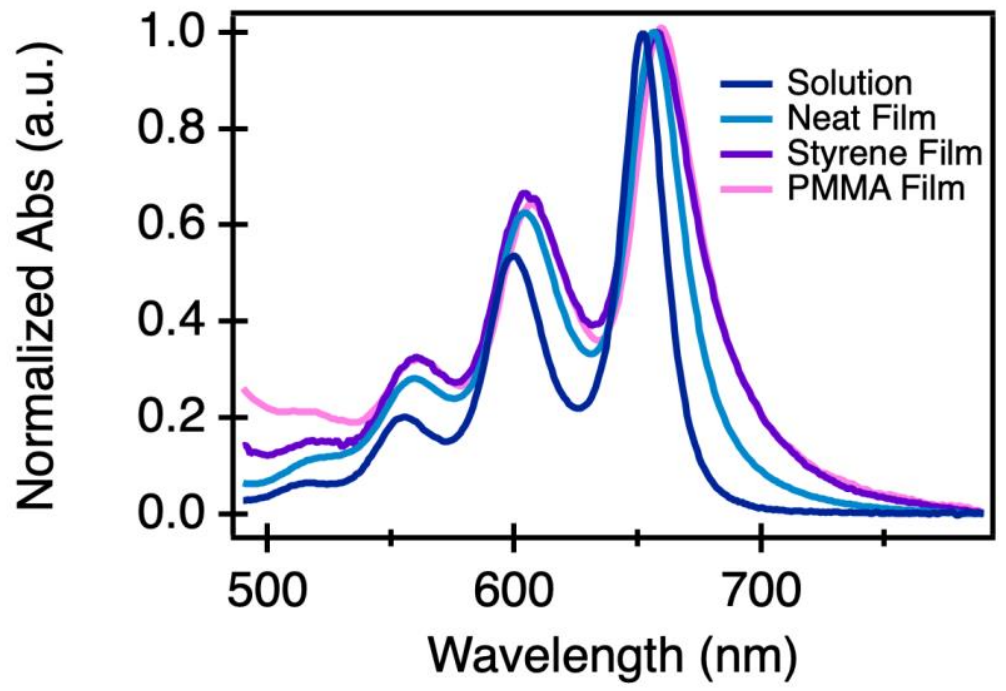

Figure S11: UV/Vis spectra of PePNo29 in solution, neat film, polystyrene matrix, and PMMA matrix. 


\section{Neutron Scattering}

\section{Instrumentation and Methods}

Small-angle neutron scattering (SANS) measurements were performed on the NGB 30m SANS instrument at the NIST Center for Neutron Research (Gaithersburg, MD). Scattered neutron intensities were collected at sample-to-detector distances of $1 \mathrm{~m}$ and $4 \mathrm{~m}$, corresponding to a range of scattering vectors $q$ from $0.01 \AA^{-1}$ to $0.4 \AA^{-1}$. The scattering vector is defined in

terms of the neutron wavelength $\lambda=6 \AA$ and scattering angle $\theta$ as $q=(4 \pi / \lambda) \sin (\theta / 2)$. Neutron measurements were corrected for empty cell scattering and background radiation and placed on an absolute scale using standard techniques. Measurements were performed at $T=25{ }^{\circ} \mathrm{C} .26 \mathrm{mg}$ of PePNo24 was dissolved in $1.5 \mathrm{~mL}$ of toluene- $\mathrm{d}_{8}(\mathrm{ca} .2 \% \mathrm{w} / \mathrm{w})$. The sample cell was covered with aluminum foil and kept dark until the measurements were taken.

SANS data were fit to a form factor describing polymer chains with excluded volume, ${ }^{6}$

$$
P(q)=\frac{1}{v U^{1 / 2 v}} \gamma\left(\frac{1}{2 v}, U\right)-\frac{1}{v U^{1 / v}} \gamma\left(\frac{1}{v}, U\right)
$$

where $v$ is an excluded volume parameter describing the conformation of the polymer chains and the variable $U=q^{2} R_{g}{ }^{2}(2 v+1)(2 v+2) / 6 . \gamma$ is the lower incomplete gamma function. $R_{g}$ is the average radius of gyration of the polymer. The excluded volume parameter $v=0.50$ for ideal polymer chains and approximately 0.6 for swollen polymer chains in solution that interact favorably with the solvent. Values of $v<0.50$ are indicative of more compact, collapsed structures.

SANS measurements from PePNo29 are shown in Figure S12 (points), along with a fit to the model above (line). The lack of an upturn in the scattering intensities at low values of $q$ implies that the polymer dissolves extremely well in toluene- $\mathrm{d}_{8}$, and that no aggregation of the chains occurs. The relatively steep slope of the curve from $q \approx 0.03 \AA^{-1}$ to $q=0.1 \AA^{-1}$ implies that the polymer chain is relatively compact as compared to most well-dissolved polymers. Analysis of the fit result finds that $R_{g}=4.2 \mathrm{~nm}$ and the excluded volume parameter $v \approx 0.3$, confirming that the polymer is compact and globule like. Interestingly, this compact conformation of polynorbornene-like polymers synthesized via ROMP has been observed in other systems. ${ }^{7}$ 


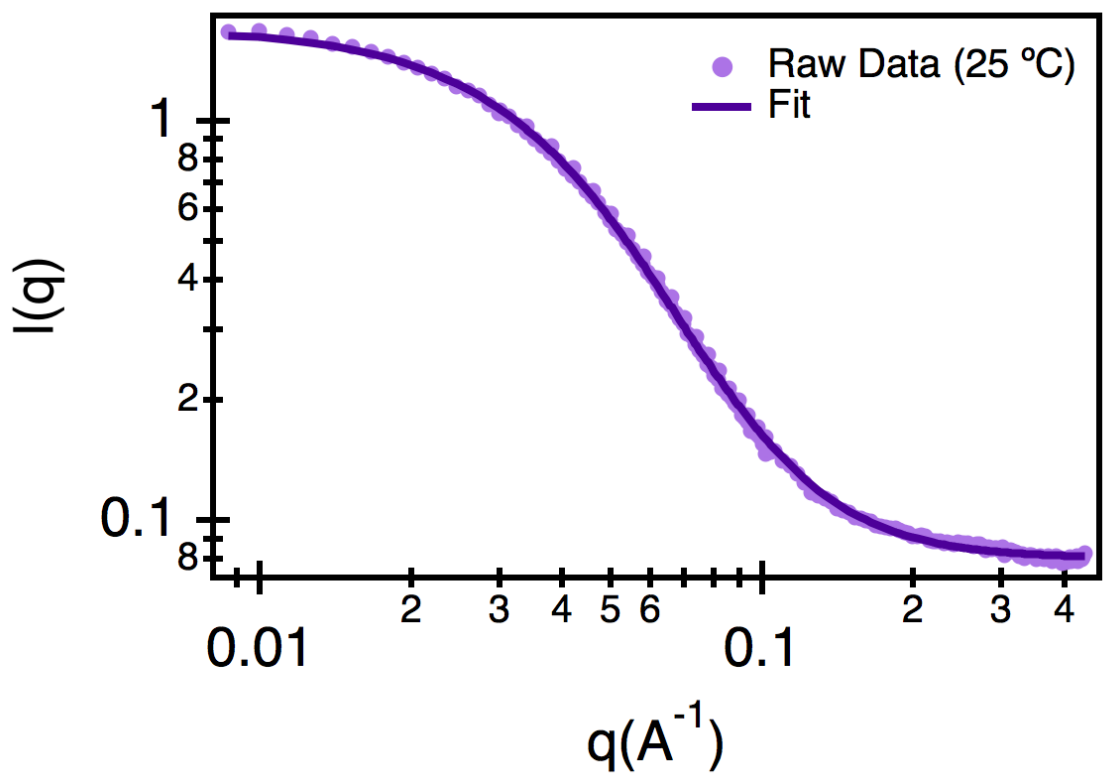

Figure S12: Small-angle neutron scattering (SANS) data taken at room temperature.

To gain additional insight into the shape of the polymer chains, the SANS data were fit to the Gunier-Porod (GP) model, ${ }^{8}$ which is an empirical scattering model for objects of arbitrary size and shape. The GP model yields a radius of gyration $\left(R_{g}\right)$ for the object, a shape parameter $s$, which describes the anisotropy of the object ( $s=0$ for spheres and $s=1$ for cylinders), and a porod exponent $m$ that describes scattering at high values of $q(m \approx 1 / v)$. The best fit of the data found $R_{g} \approx 3 \mathrm{~nm}$, which is close to the value obtained from the polymer with excluded volume model. The value of $s=0.25$ indicates that the polymer globules are slightly anisotropic and the value of the Porod exponent $m=2.74$ confirms that the chains form globular-like objects in solution. These values are in agreement with SANS measurements of other polynorbornene-like polymers, which show similar values for the anisotropy and Porod exponents. ${ }^{7,9}$ Using a flexible cylinder form factor, and constraining the length of the cylinder to be approximately the $R_{g}$ of the polymer measured, we estimate that the persistence length is on the order of $5 \mathrm{~nm}$, which is half of the value measured for bottlebrush polymers composed of similar monomer units and implies that on the order of 5 monomers are present in each persistence length. ${ }^{9}$ 


\section{Synthetic Information}

A. General Methods

Atmosphere

All reactions were carried out under argon in oven dried glassware unless otherwise stated.

\section{Materials and Solvents}

All reagents/solvents were purchased from Alfa Aesar ${ }^{\circledR}$, Sigma-Aldrich $^{\circledR}$, Acros $\operatorname{organics}^{\circledR}$, TCI America ${ }^{\circledR}$, Mallinckrodt ${ }^{\circledR}$, and Oakwood ${ }^{\circledR}$. They were used without further purification. A Schlenk manifold with purification columns packed with activated alumina and support copper catalyst (Glass Contour, Irvine, CA) was used to obtain dry solvents. The polymerization catalyst, $\left(\mathrm{IMesH}_{2}\right)(\mathrm{Cl})_{2}\left(\mathrm{C}_{5} \mathrm{H}_{5} \mathrm{~N}\right)_{2} \mathrm{Ru}=\mathrm{CHPh}$ (Grubbs III), was synthesized according to literature procedure. ${ }^{10}$ 2-Bromo-6,13-Bis(triisopropylsilylethynyl)pentacene was synthesized according to literature procedure. ${ }^{1,11}$

\section{Instrumentation}

Bruker $400 \mathrm{MHz}$ and $500 \mathrm{MHz}$ spectrometers were used for ${ }^{1} \mathrm{H}-\mathrm{NMR}$, and Bruker 100 $\mathrm{MHz}$ and $125 \mathrm{MHz}$ spectrometers were used for ${ }^{13} \mathrm{C}-\mathrm{NMR}$. Chemical shifts are reported in ppm $(\delta)$ along with the integration values. The coupling constants $(\mathrm{J})$ are reported in hertz $(\mathrm{Hz})$, and standard multiplicity abbreviations were used: s (singlet), $\mathrm{d}$ (doublet), $\mathrm{t}$ (triplet), and $\mathrm{m}$ (multiplet). XEVO G2-XS Waters ${ }^{\circledR}$ equipped with a QTOF detector with multiple inlet and ionization capabilities including electrospray ionization (ESI), atmospheric pressure chemical ionization (APCI), and atmospheric solids analysis probe (ASAP) were used to take mass spectral data 
B. General protocol for the synthesis of pentacene polymers:

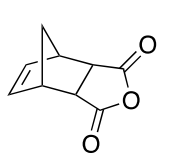

1

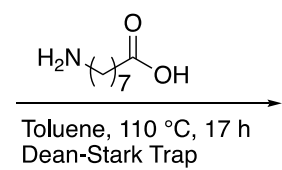

Dean-Stark Trap

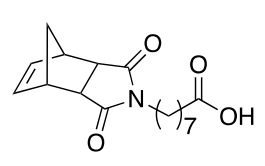

2
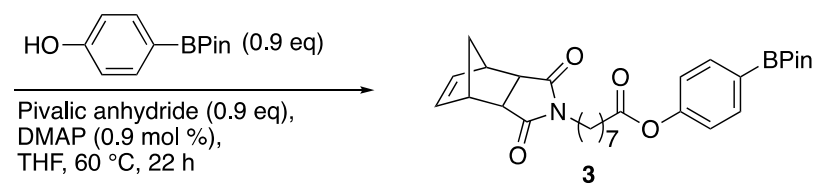
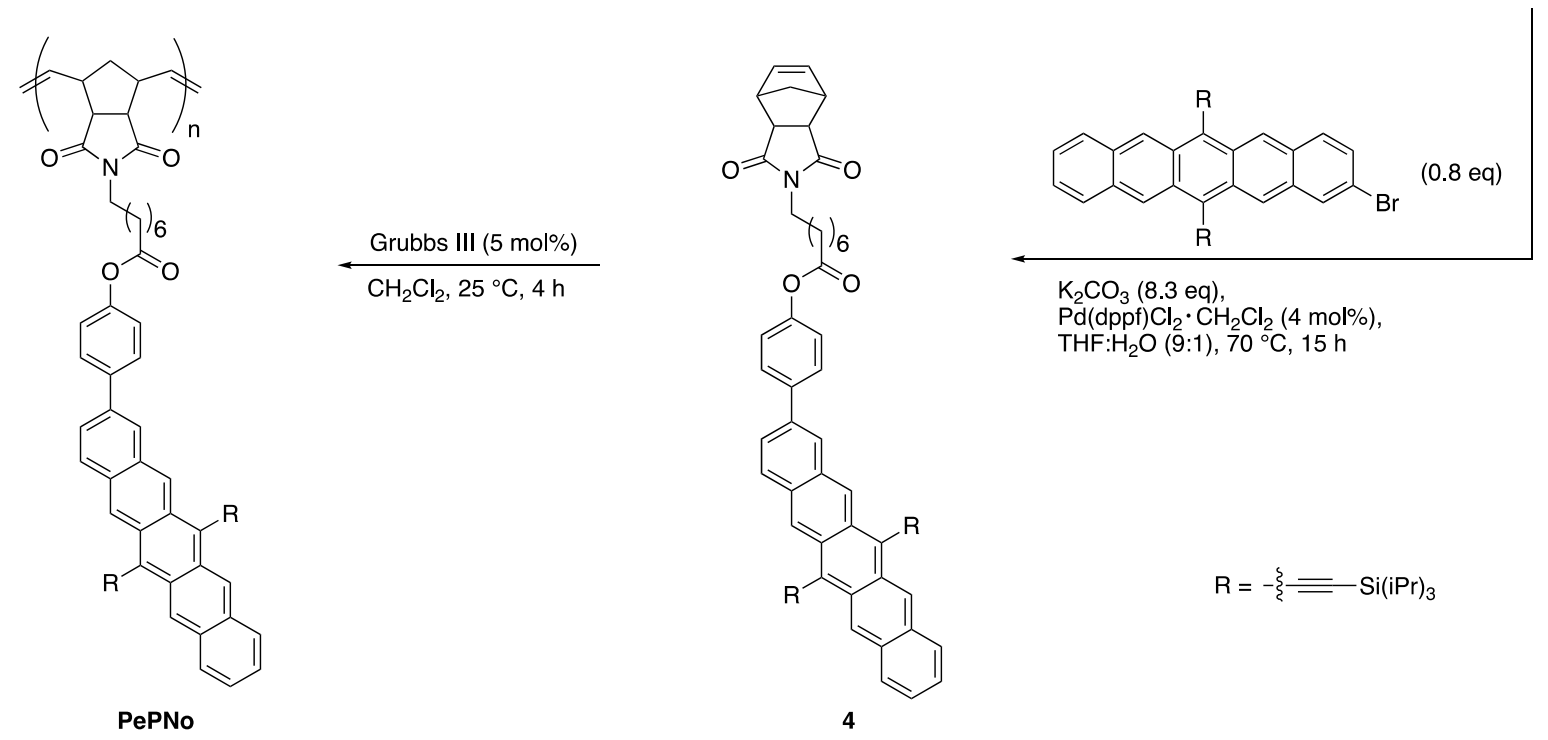

$\mathrm{Pd}(\mathrm{dppf}) \mathrm{Cl}_{2} \cdot \mathrm{CH}_{2} \mathrm{Cl}_{2}(4 \mathrm{~mol} \%)$

THF: $\mathrm{H}_{2} \mathrm{O}(9: 1), 70^{\circ} \mathrm{C}, 15 \mathrm{~h}$

$\mathrm{R}=-\xi \overline{\mathrm{Si}}(\mathrm{PPr})_{3}$

C. Synthetic details:

Synthesis of $\mathbf{2}$

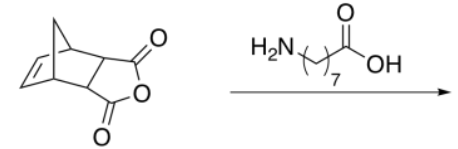

1

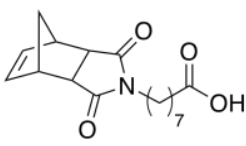

2

A slurry of cis-5-norbornene-exo-2,3-dicarboxylic anhydride (1) (16.8 mmol, 2.76 g, 1 equiv.) and 8-aminooctanoic acid (17.6 mmol, $2.81 \mathrm{~g}, 1.05$ equiv.) in dry toluene ( $85 \mathrm{~mL})$ were heated to reflux in a round bottom flask fitted with a Dean-Stark trap and reflux condenser. The reaction refluxed for $17 \mathrm{~h}$. The reaction came to room temperature and was washed three times with $1 \mathrm{M}$ $\mathrm{HCl}$, three times with brine, dried over $\mathrm{MgSO}_{4}$, filtered, and concentrated on the rotary evaporator to give a white solid (quantitative yield). The product was used without further purification. 
${ }^{1} \mathrm{H}-\mathrm{NMR}\left(500 \mathrm{MHz}, \mathrm{CDCl}_{3}, \delta \mathrm{ppm}\right): 6.28(\mathrm{~s}, 2 \mathrm{H}), 3.44(\mathrm{t}, J=10 \mathrm{~Hz}, 2 \mathrm{H}), 3.26(\mathrm{~s}, 2 \mathrm{H}), 2.66$ (s, 2H), $2.33(\mathrm{t}, J=7.5 \mathrm{~Hz}, 2 \mathrm{H}), 1.63-1.49(\mathrm{~m}, 5 \mathrm{H}), 1.32(\mathrm{~m}, 6 \mathrm{H}), 1.22-1.20(\mathrm{~m}, 1 \mathrm{H})$.

${ }^{13} \mathrm{C}-\mathrm{NMR}$ (125 MHz, $\left.\mathrm{CDCl}_{3}, \delta \mathrm{ppm}\right):$ 178.3, 138.0, 47.9, 45.3, 42.9, 38.8, 34.0, 34.0, 29.0, 28.8, 27.8, 26.9, and 24.7.

MS (ESI): Calculated ([M-H]'): 304.1549; Observed: 304.1547.

Synthesis of $\mathbf{3}$
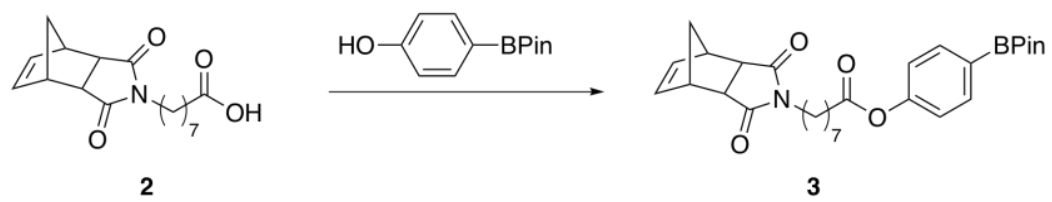

Dry tetrahydrofuran (THF) (15 mL) was added to a round bottom flask containing 2 (16.4 mmol, $5 \mathrm{~g}, 1.1$ equiv.), 4-hydroxyphenylboronic acid pinacol ester (14.9 mmol, $3.28 \mathrm{~g}, 1.0$ equiv.), and 4-dimethylaminopyridine (DMAP) (0.149 mmol, $18 \mathrm{mg}, 0.01$ equiv.). The mixture stirred until nearly dissolved. Pivalic anhydride (16.4 mmol, $3.05 \mathrm{~g}, 3.3 \mathrm{~mL} 1.1$ equiv.) was added to the solution. The reaction stirred at $60{ }^{\circ} \mathrm{C}$ for $22 \mathrm{hrs} . \mathrm{H}_{2} \mathrm{O}(5 \mathrm{~mL})$ was added, and the reaction stirred for $1 \mathrm{~h}$. Dichloromethane (DCM) was added to the reaction mixture. The solution was washed three times with sat. sodium bicarbonate, one time with brine, dried over $\mathrm{MgSO}_{4}$, filtered, and concentrated on a rotary evaporator. A silica column was run in $20 \%$ ethyl acetate in hexanes, which afforded a white solid (70\% yield).

${ }^{1} \mathrm{H}-\mathrm{NMR}\left(500 \mathrm{MHz}, \mathrm{CDCl}_{3}, \delta \mathrm{ppm}\right): 7.82(\mathrm{~d}, J=8.3 \mathrm{~Hz}, 2 \mathrm{H}), 7.07(\mathrm{~d}, J=8.3 \mathrm{~Hz}, 2 \mathrm{H}), 6.28(\mathrm{~s}$, 2H), $3.46(\mathrm{t}, J=7.5 \mathrm{~Hz}, 2 \mathrm{H}), 3.27(\mathrm{~s}, 2 \mathrm{H}), 2.66(\mathrm{~s}, 2 \mathrm{H}), 2.54(\mathrm{t}, J=7.5 \mathrm{~Hz}, 2 \mathrm{H}), 1.73(\mathrm{~m}, 2 \mathrm{H})$, $1.55(\mathrm{~m}, 2 \mathrm{H}$, overlapping water peak), $1.33(\mathrm{~m}, 19 \mathrm{H}), 1.22(\mathrm{~m}, 1 \mathrm{H})$.

${ }^{13} \mathrm{C}-\mathrm{NMR}\left(125 \mathrm{MHz}, \mathrm{CDCl}_{3}, \delta \mathrm{ppm}\right): 178.2,172.0,153.4,138.0,136.3,121.1,84.0,48.0,45.3$, $42.9,38.8,34.5,29.0,28.9,27.8,26.9,25.0$, and 24.9.

MS (ESI): Calculated ([M+NH$\left.]^{+}\right)$: 525.3141; Observed: 525.3151. 
Synthesis of 4

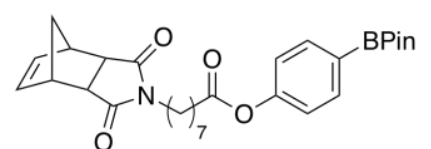

3

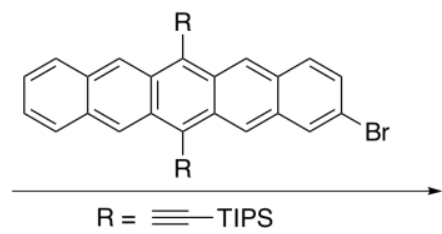

$\mathrm{R}=\equiv$ TIPS

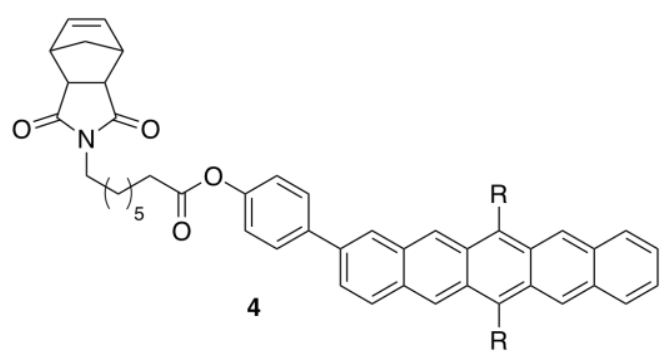

To a two-neck round bottom flask was added 3 (1.94 mmol, $0.98 \mathrm{~g}, 1.2$ equiv.), 2-bromo-6,13bis(triisopropylsilylethynyl)pentacene (1.62 mmol, $1.11 \mathrm{~g}, 1$ equiv.), and $\mathrm{Pd}(\mathrm{dppf}) \mathrm{Cl}_{2} \bullet \mathrm{CH}_{2} \mathrm{Cl}_{2}$ (0.081 mmol, $66 \mathrm{mg}, 0.05$ equiv.) followed by dry THF $(35 \mathrm{~mL})$. A degassed solution of $\mathrm{K}_{2} \mathrm{CO}_{3}$ (16.2 mmol, $2.24 \mathrm{~g}, 10$ equiv.) in $\mathrm{H}_{2} \mathrm{O}(3.9 \mathrm{~mL})$ was subsequently added. The reaction stirred for $18 \mathrm{~h}$ at $70{ }^{\circ} \mathrm{C}$. The reaction mixture came to room temperature and the water was pipetted out before concentrating on the rotary evaporator. The crude mixture was purified by column chromatography in chloroform affording a blue solid (60\% yield).

${ }^{1} \mathrm{H}-\mathrm{NMR}\left(400 \mathrm{MHz}, \mathrm{CDCl}_{3}, \delta \mathrm{ppm}\right): 9.37(\mathrm{~m}, 4 \mathrm{H}), 8.14(\mathrm{~m}, 1 \mathrm{H}), 8.09(\mathrm{~d}, J=9.2 \mathrm{~Hz}, 1 \mathrm{H}), 8.02$ $(\mathrm{dd}, J=6.6,3.1 \mathrm{~Hz}, 2 \mathrm{H}), 7.84-7.80(\mathrm{~m}, 2 \mathrm{H}), 7.71(\mathrm{dd}, J=8.9,1.5 \mathrm{~Hz}, 1 \mathrm{H}), 7.47-7.43(\mathrm{~m}$, 2H), $7.28-7.25(\mathrm{~m}, 2 \mathrm{H}), 6.31(\mathrm{~m}, 2 \mathrm{H}), 3.55-3.51(\mathrm{~m}, 2 \mathrm{H}), 3.53(\mathrm{t}, J=7.5 \mathrm{~Hz} 2 \mathrm{H}), 2.70(\mathrm{~m}$, $2 \mathrm{H}), 2.63(\mathrm{t}, J=7.5 \mathrm{~Hz}, 2 \mathrm{H}), 1.71-1.22(\mathrm{~m}, 57 \mathrm{H}$, overlapping water peak)

${ }^{13} \mathrm{C}-\mathrm{NMR}\left(125 \mathrm{MHz}, \mathrm{CDCl}_{3}, \delta \mathrm{ppm}\right): 178.27,172.44,150.58,138.65,137.98,137.67,132.47$, 131.53, 131.08, 130.89, 130.77, 129.56, 128.82, 128.45, 126.73, 126.48, 126.31, 126.20, 126.07, $122.24,118.60,118.43,107.48,47.97,45.33,42.89,38.81,34.51,29.08,28.92,27.86,26.92$, $24.99,19.18,19.16$, and 11.84 .

MS (ESI): Calculated $\left([\mathrm{M}+\mathrm{H}]^{+}\right):$1018.5626; Observed: 1018.5595. 

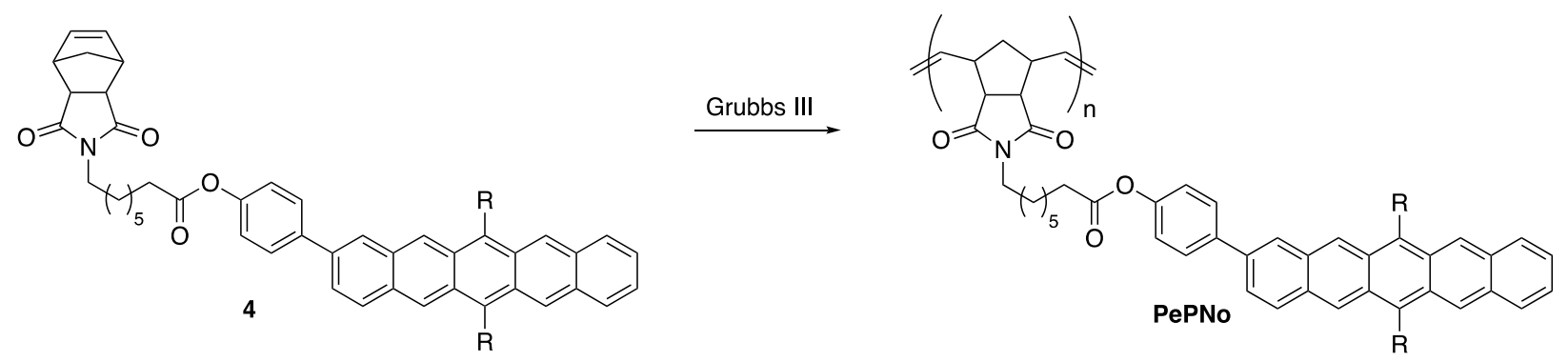

To a solution of 4 ( $0.015 \mathrm{mmol}, 15 \mathrm{mg}, 1.0$ equiv. $)$ in dry $\mathrm{CH}_{2} \mathrm{Cl}_{2}$ was added varying amounts of Grubbs III in dry $\mathrm{CH}_{2} \mathrm{Cl}_{2}$ for a final concentration of $0.02 \mathrm{M}$ (see Table S2). The reaction stirred for $4 \mathrm{~h}$ at room temperature. Ethyl vinyl ether was added to quench the reaction. After stirring an hour, the solution was precipitated into $-78{ }^{\circ} \mathrm{C} \mathrm{MeOH}$ and filtered. The filtrate was discarded, and the polymer was dissolved in $\mathrm{CH}_{2} \mathrm{Cl}_{2}$, collected, and concentrated to give a blue solid (quantitative yield).

${ }^{1} \mathrm{H}-\mathrm{NMR}\left(400 \mathrm{MHz}, \mathrm{CDCl}_{3}, \delta \mathrm{ppm}\right): 9.25$ (m, 3H), 8.02-7.92 (m, 4H), 7.71-7.59 (m, 3H), 7.35 (m, 2H), $7.17(\mathrm{~m}, 2 \mathrm{H}), 5.76(\mathrm{~m}, 1 \mathrm{H}), 5.46(\mathrm{~m}, 1 \mathrm{H}), 3.44(\mathrm{~m}), 3.29(\mathrm{~m}), 2.99(\mathrm{~m}), 2.54(\mathrm{~m}), 1.74$ (m), $1.53(\mathrm{~m}), 1.33(\mathrm{~m}), 0.88(\mathrm{~m}), 0.61(\mathrm{~m})$. 


\section{NMR Spectra}

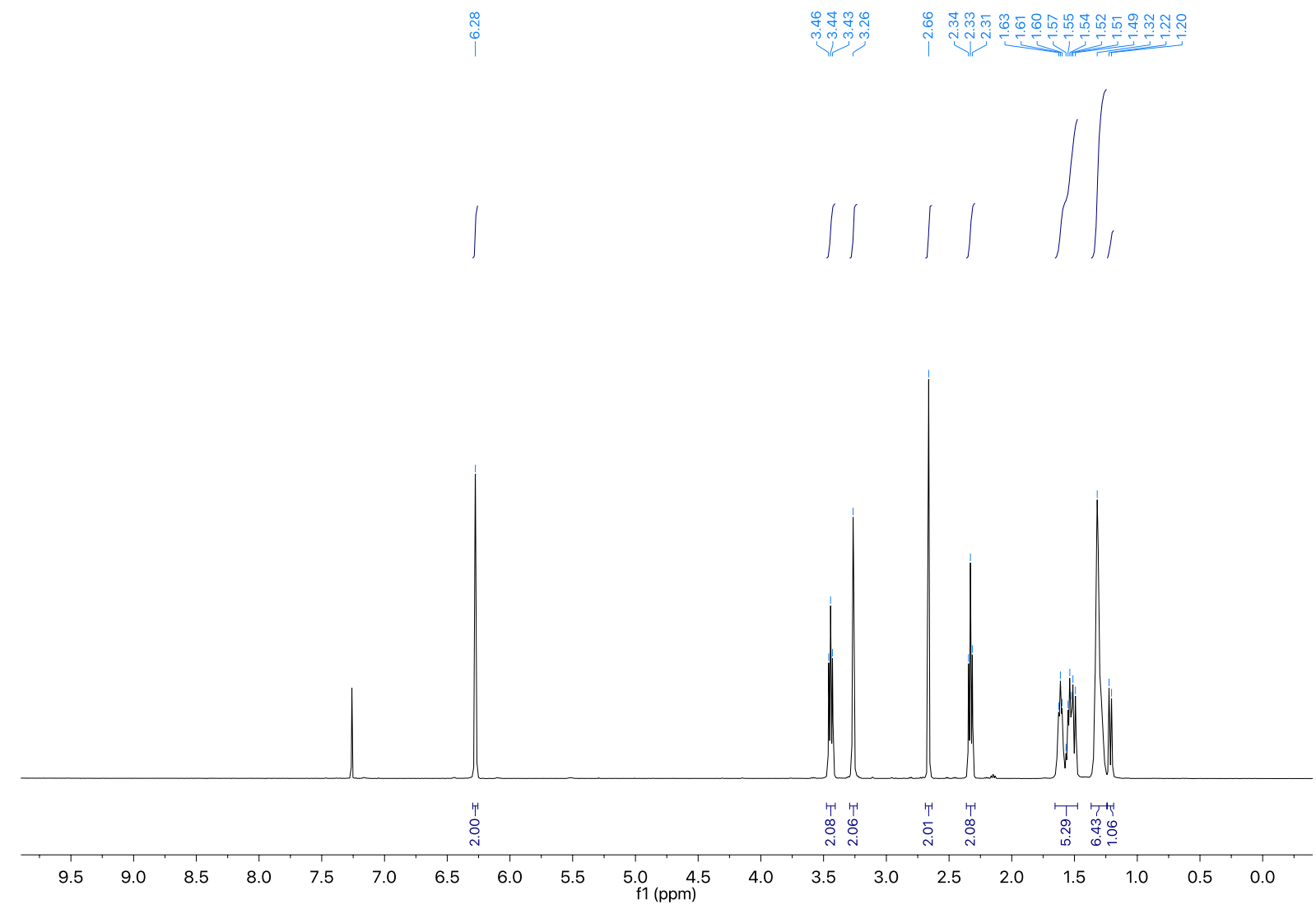

Figure S13: ${ }^{1} \mathrm{H}-\mathrm{NMR}$ spectrum of 2. 


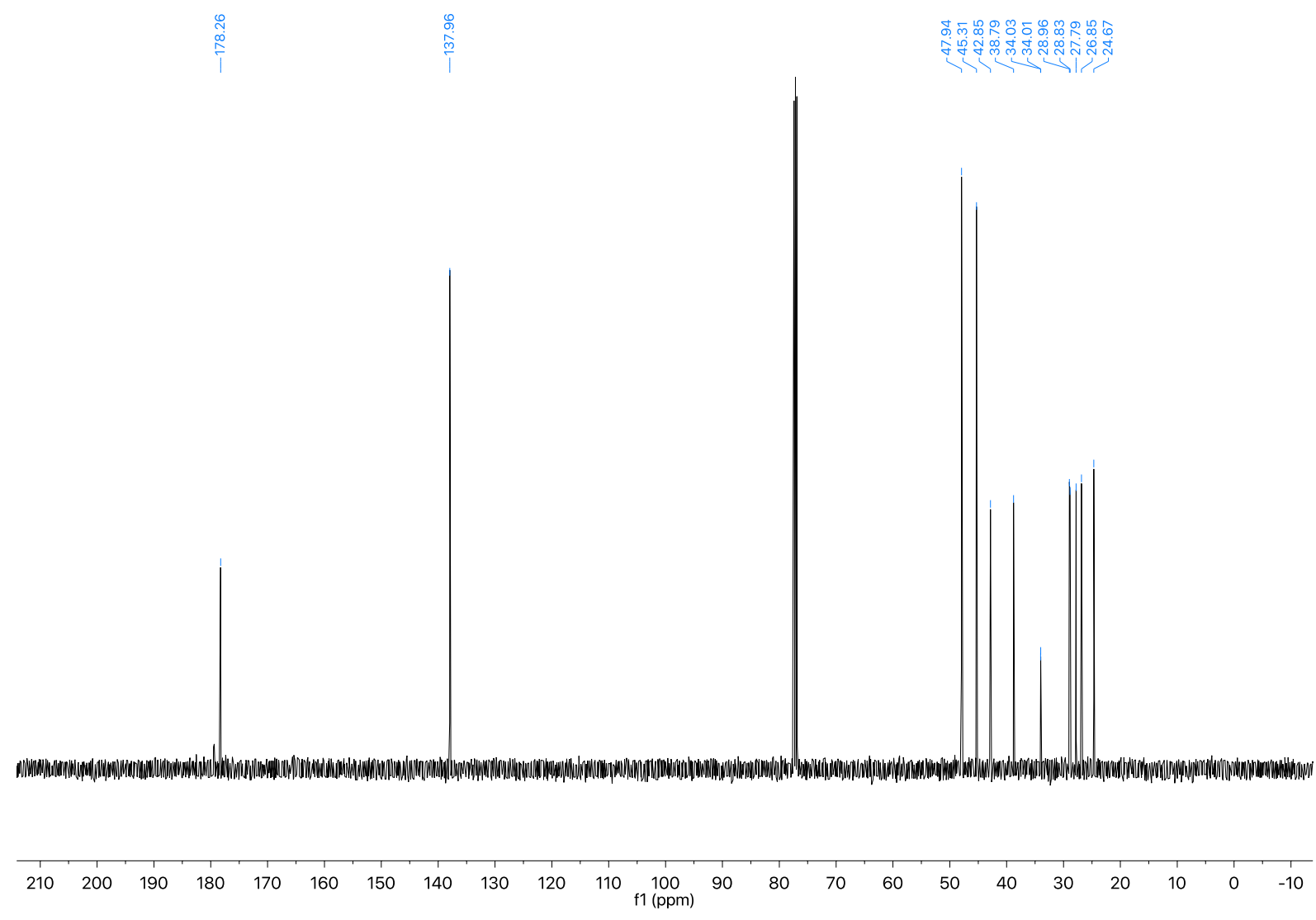

Figure S14: ${ }^{13} \mathrm{C}-\mathrm{NMR}$ spectrum of 2. 


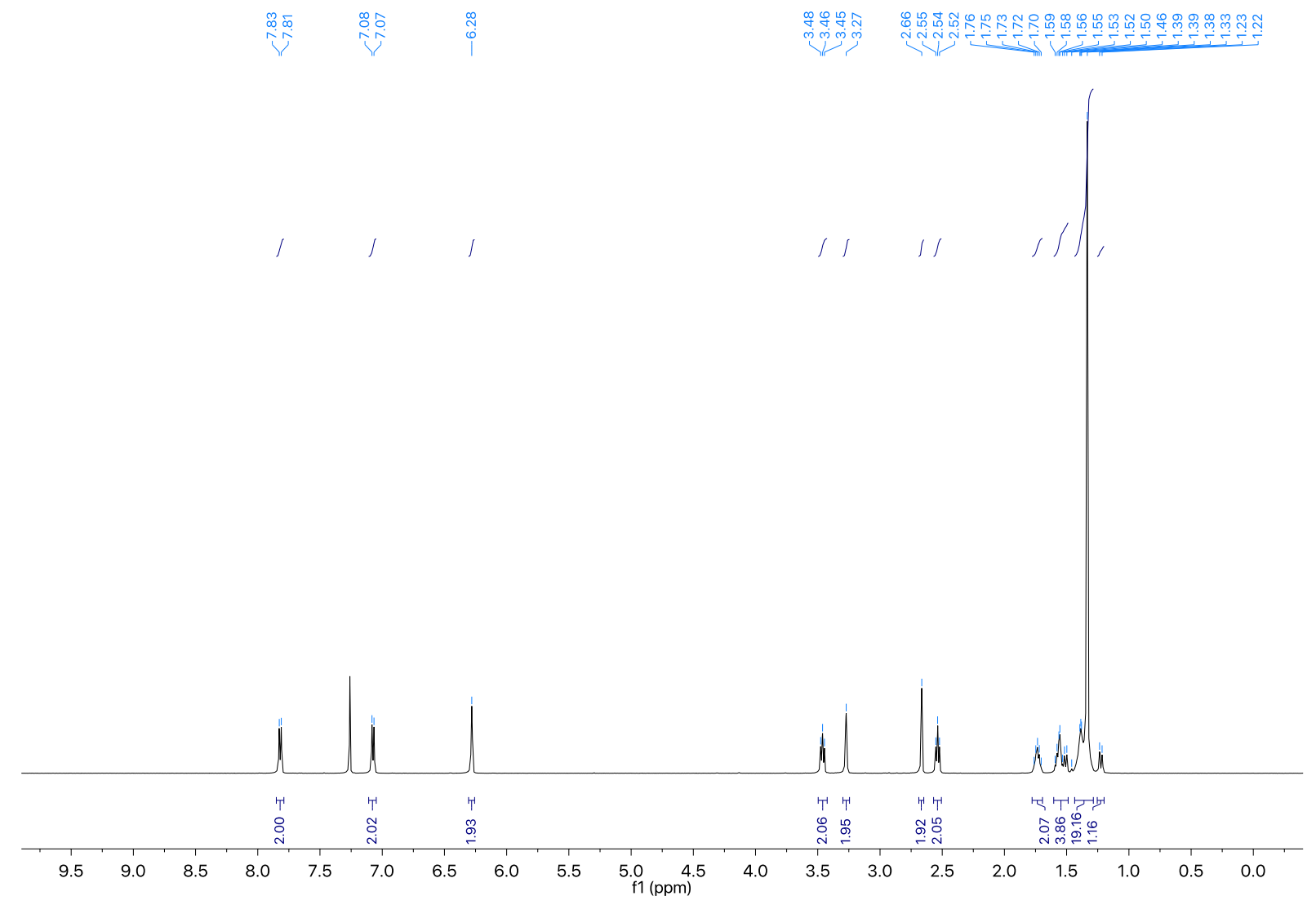

Figure S15: ${ }^{1} \mathrm{H}-\mathrm{NMR}$ spectrum of 3 . 


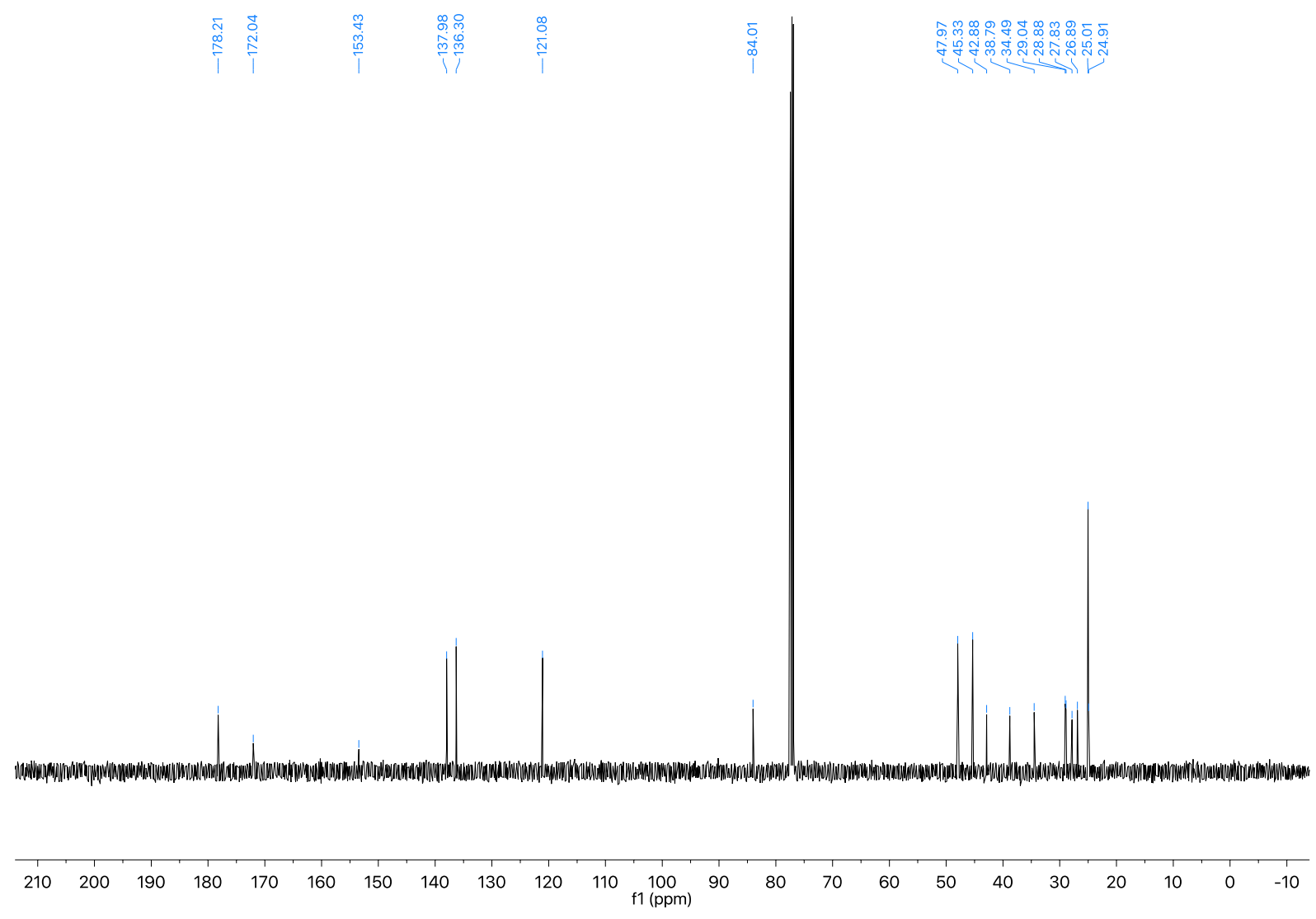

Figure S16: ${ }^{13} \mathrm{C}-\mathrm{NMR}$ spectrum of $\mathbf{3}$. 


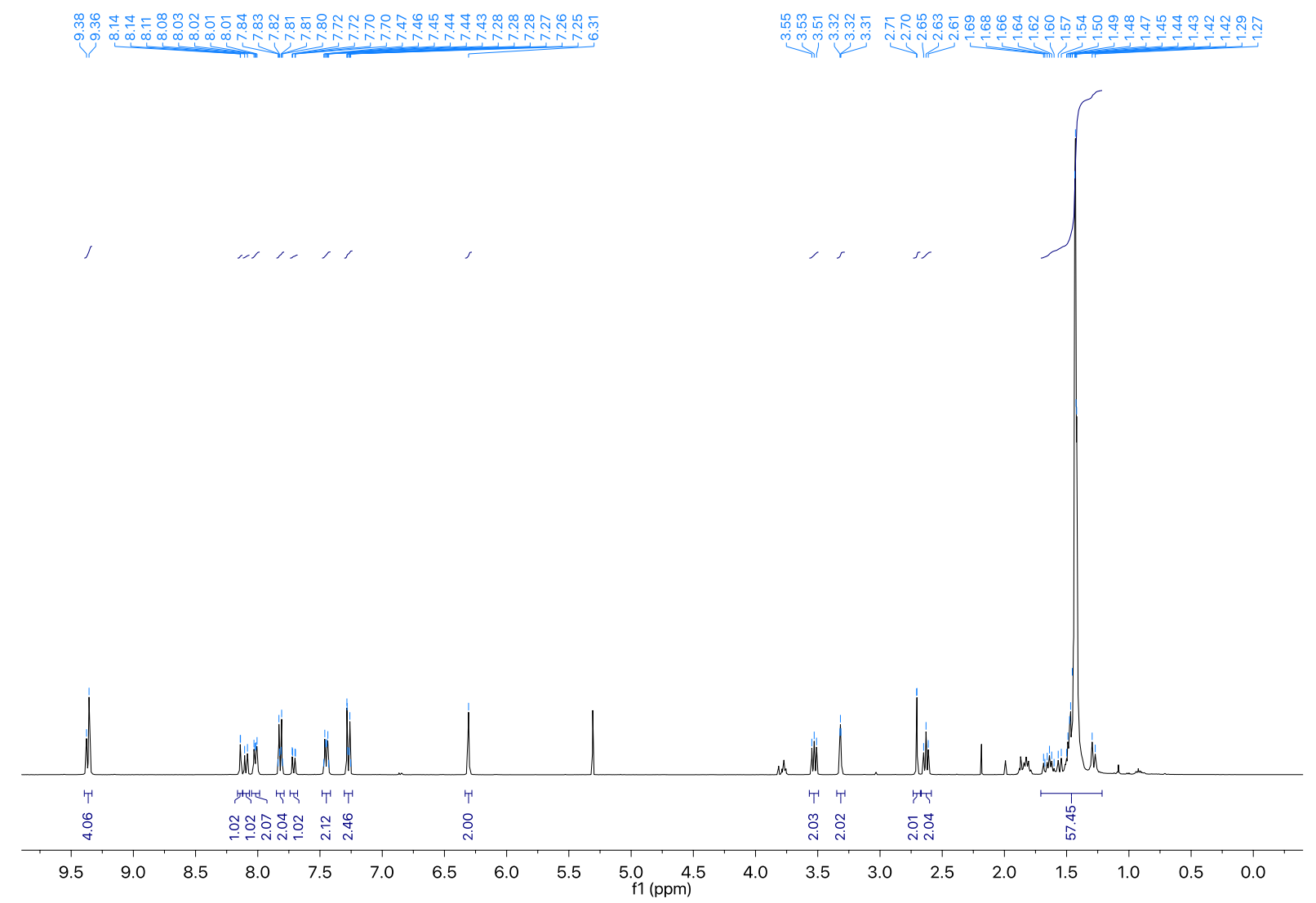

Figure S17: ${ }^{1} \mathrm{H}-\mathrm{NMR}$ spectrum of 4 . 


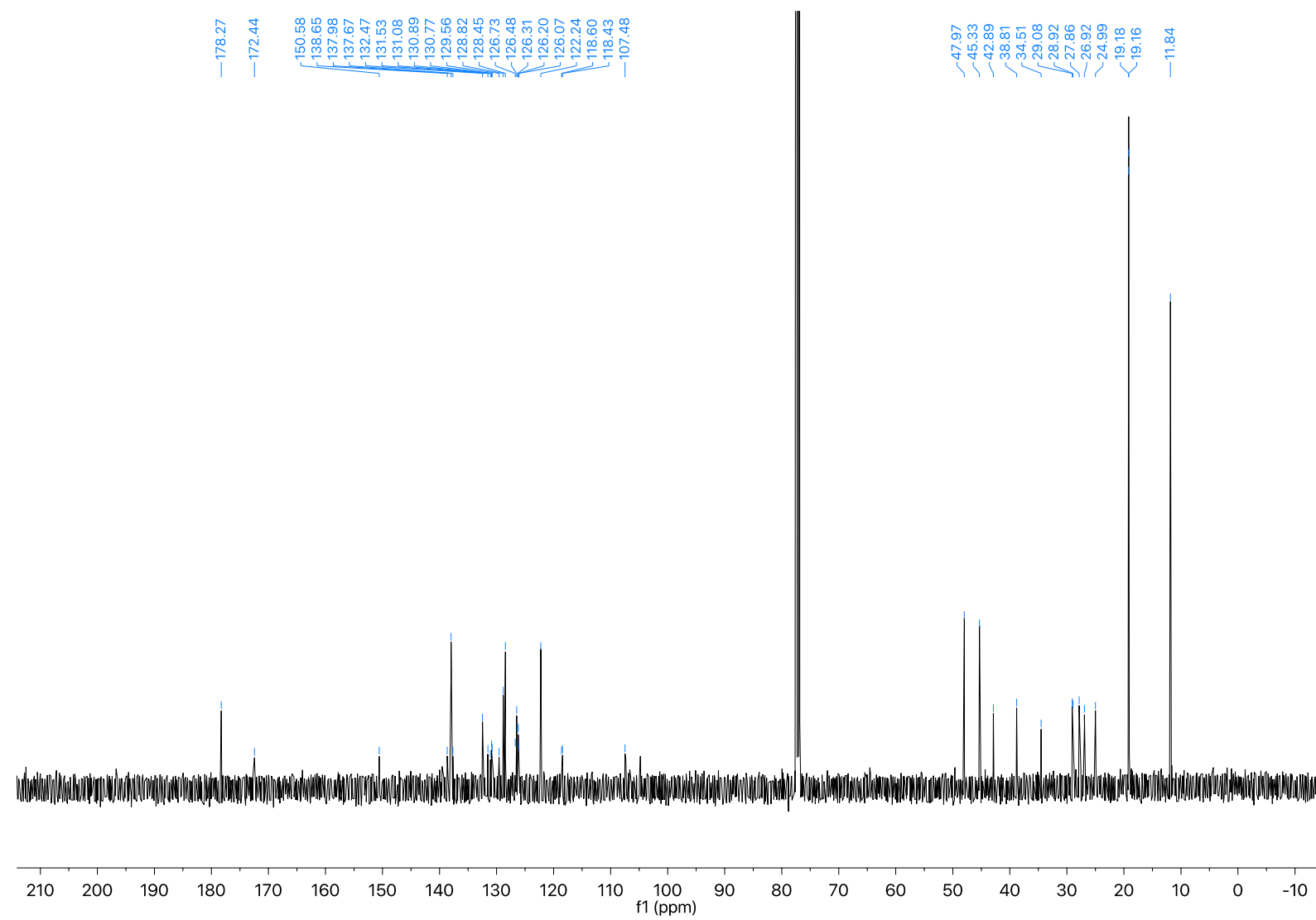

Figure S18: ${ }^{13} \mathrm{C}-\mathrm{NMR}$ spectrum of 4 . 


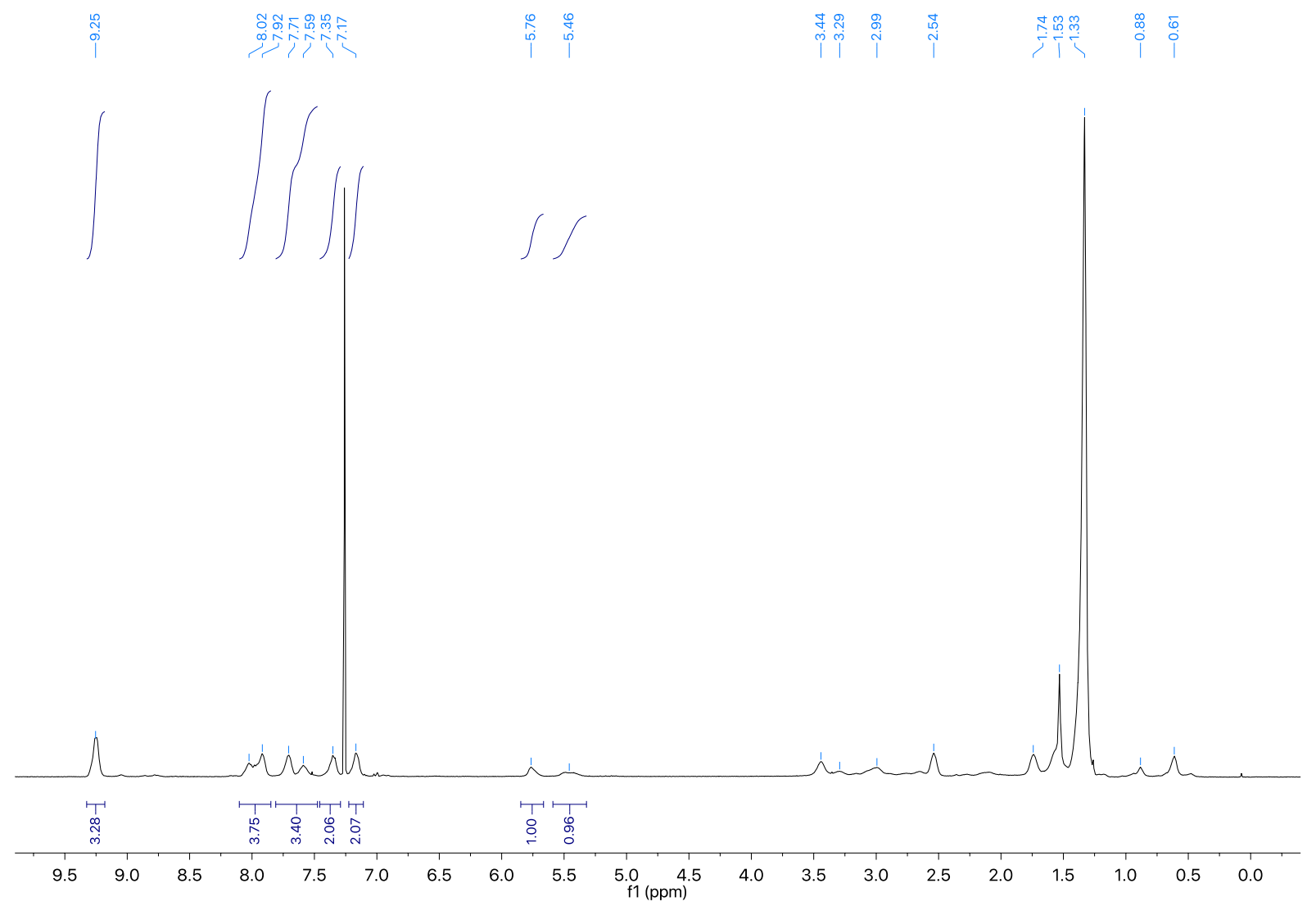

Figure S19: ${ }^{1} \mathrm{H}-\mathrm{NMR}$ spectrum of PePNo24. 


\section{Molecular Weight Data}

\section{Instrumentation}

Bruker UltrafleXtreme MALDI-TOF/TOF instrument was used for MALDI-TOF measurements. Gel permeation chromatography (GPC) was taken on a Waters Alliance 2695 separation module equipped with a PL-aqua gel-OH 8-micron Mixed-M column (300 x $7.5 \mathrm{~mm})$ with a Waters 2998 Photodiode Array Detector and a Waters 2414 Refractrometer Detector with an elution rate of $1 \mathrm{~mL} / \mathrm{min}$. Measurements were taken in dibutylhydroxytoluene stabilized tetrahydrofuran (THF). Molecular weight data and dispersities were determined from monodisperse polystyrene standards.

Table S2: Catalyst loading from the different polymerization reactions and molecular weight data from the GPC.

\begin{tabular}{|c|c|c|c|c|c|}
\hline & $\begin{array}{c}\text { Theoretical } \\
\text { Repeat Units }\end{array}$ & $\begin{array}{c}\text { Mol \% } \\
\text { Grubbs III }\end{array}$ & Mn (kg/mol) & Mw (kg/mol) & Đ \\
\hline Monomer & - & - & 1.1 & 1.2 & - \\
\hline PePNo8 & 5 & 0.2 & 7.8 & 8.7 & 1.13 \\
\hline PePNo10 & 10 & 0.1 & 9.7 & 11 & 1.11 \\
\hline PePNo16 & 20 & 0.05 & 16 & 18 & 1.15 \\
\hline PePNo24 & 40 & 0.025 & 24 & 33 & 1.42 \\
\hline PePNo29 & 40 & 0.025 & 29 & 44 & 1.53 \\
\hline PePNo33 & 60 & 0.017 & 33 & 52 & 1.59 \\
\hline
\end{tabular}

The MALDI-TOF spectrum of PePNo8 is shown in Figure S20. We see a distribution of molecular weights centered on the molecular weight we would expect for PePNo8. It should be noted however that MALDI-TOF is not a quantitative technique and conclusions cannot be drawn about the relative amounts of each oligomer based on the spectrum. ${ }^{12}$ 


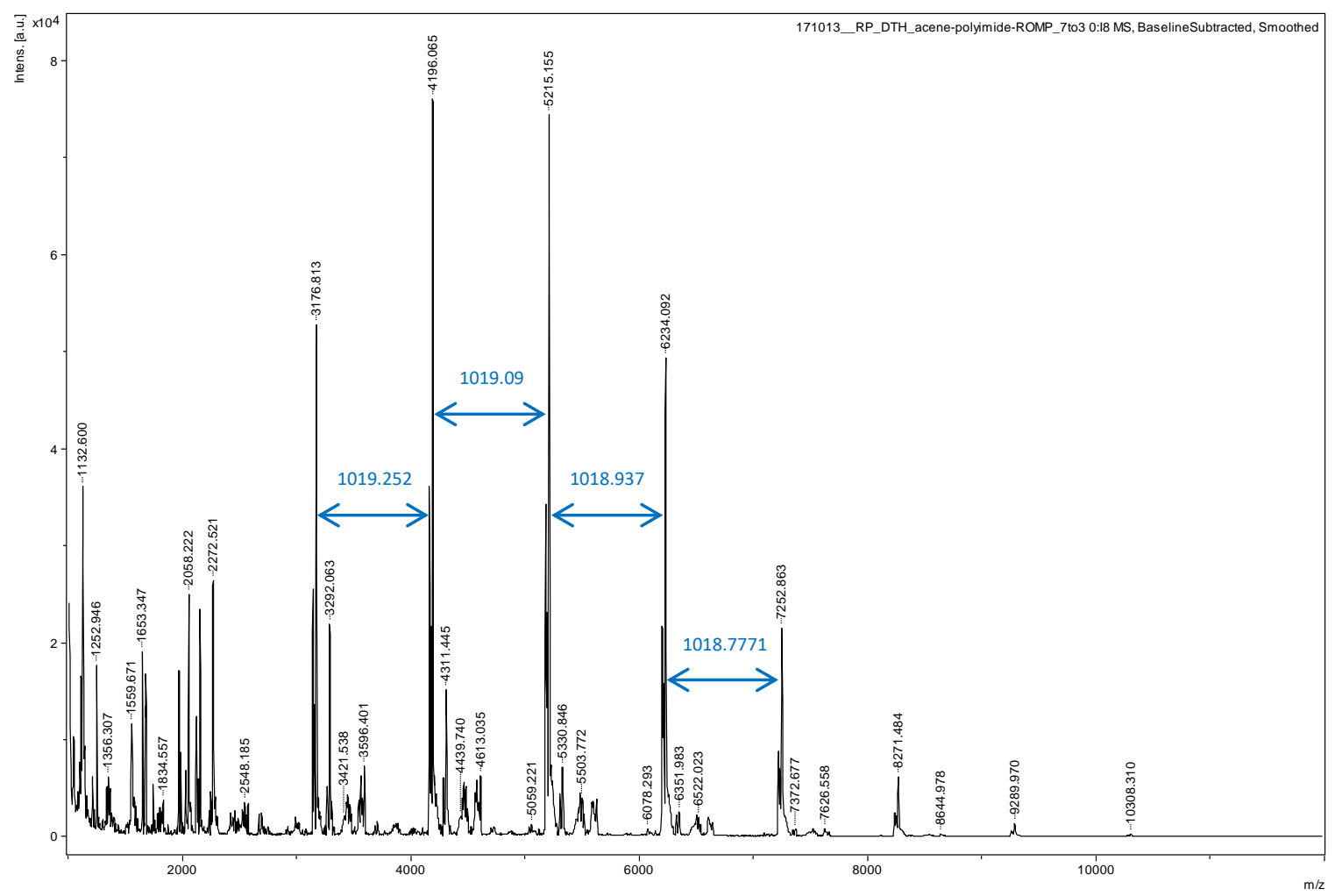

Figure S20: MALDI-TOF spectrum of PePNo8 with baseline correction. 


\section{Yield Calculation}
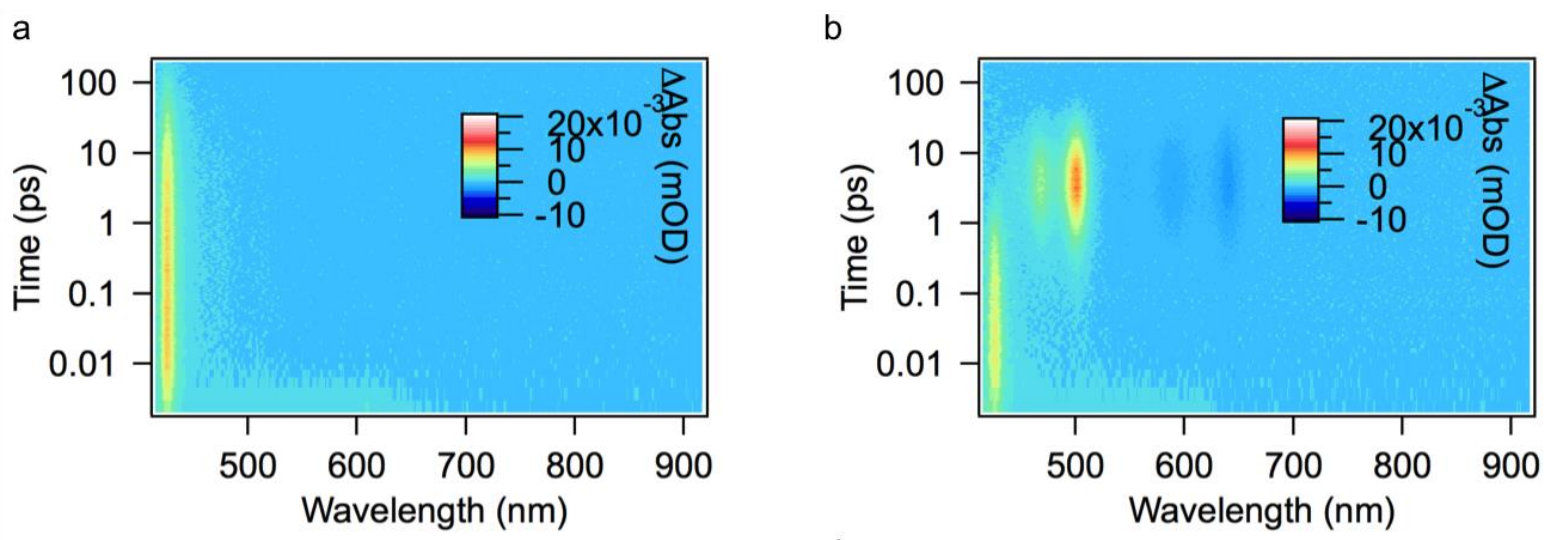

C

d
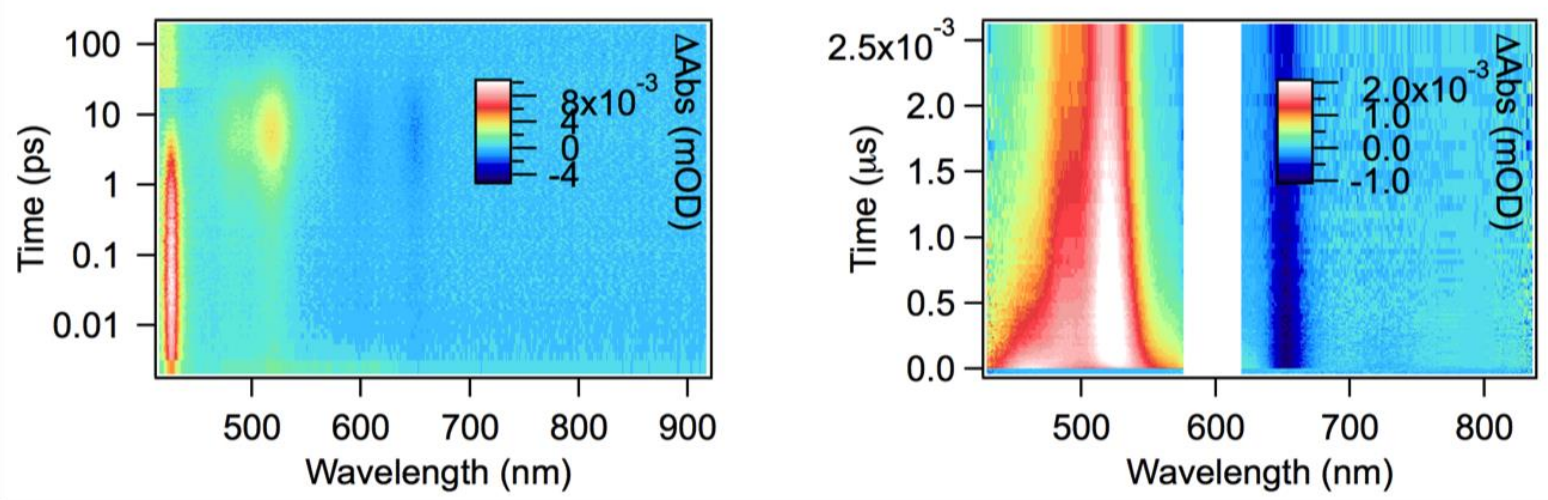

Figure S21: The necessary plots for the sensitization yield determination. (a) TA spectrum of anthracene in toluene. (b) TIPS pentacene sensitization TA spectrum. (c) Sensitization of PePNo33. (d) TA spectrum of PePNo33 $(200 \mu \mathrm{W})$.

The singlet fission yield is calculated using establish methods. Briefly, the molar extinction coefficients for the singlet ground state bleach and triplet excited state absorption signals are independently determined. The singlet fission yield is then determined using these molar extinction coefficients to determine the ratio of the triplet concentration to the concentration of initial singlet excitons after singlet fission. We estimate the error of this approach to be $+/-20 \%$. The following calculation is specific for PePNo33, though other polymers give a similar result.

\section{Determination of $\varepsilon_{S}^{P e p N o}$}

The molar extinction coefficient for the photoexcited singlet exciton $\left(\varepsilon_{S}^{P e p N o}\right)$ is estimated by populating the polymers with a known quantity of singlet excitons and measuring the 
corresponding ground state bleach signal. An automated laser stabilization procedure was used to ensure constant excitation fluence over the course of the measurement.

Excitation pump pulse $=600 \mathrm{~nm}$, power stabilization @ $0.08 \mathrm{~mW}$

Spot size $=3.3 \times 10^{-3} \mathrm{~cm}^{2}$

Fluence $=1.47 \times 10^{14}$ photons $/ \mathrm{cm}^{2}$

PePNo33 Absorbance $(\mathrm{A})=0.183$

PePNo33 Exciton Density/Path Length $(n / l)=$ Incident Fluence $*\left(1-10^{-\mathrm{A}}\right)$

$$
=1.47 \times 10^{14} \text { photons } / \mathrm{cm}^{2} *\left(1-10^{-0.183}\right)=5.1 \times 10^{13} \text { excitons } / \mathrm{cm}^{-2}
$$

PePNo33 $\triangle \mathrm{A}=2 \mathrm{mOD}$

$\varepsilon_{S}^{P e p N o}=\left(\Delta \mathrm{A} * N_{A} * 1000\right) /(n / l)=\left(0.002 * 6.02 \times 10^{23} * 1000\right) /\left(5.1 \times 10^{13}\right)=23,615 \mathrm{M}^{-1} \mathrm{~cm}^{-1}$

\section{Determination of $\varepsilon_{T}^{\text {anth }}$ for Anthracene in Toluene}

The molar extinction coefficient for the anthracene triplet exited state absorption signal for anthracene is determined via triplet sensitization of TIPS-Pentacene monomer. The singlet molar extinction coefficient for TPc in toluene $\varepsilon_{g s b}^{T P c}$ has been previously determined to be 21,000 $M^{-1} \mathrm{~cm}^{-1}$. By measuring the triplet transfer efficiency from anthracene to TPc using a kinetic analysis, $\varepsilon_{T}^{\text {anth }}$ can be directly computed.

The anthracene triplet lifetime $\tau_{T 1}^{\text {anth }}$ in toluene from fitting after direct photoexcitation of 20 $\mathrm{mM}$ anthracene in toluene: $25 \mu \mathrm{s}$.

In a toluene solution of $20 \mathrm{mM}$ anthracene and $50 \mu \mathrm{M} \mathrm{TPc}$, the collisional triplet transfer time constant from anthracene to TPc $\tau_{t t}=2 \mu \mathrm{s}$. The subsequent TPc triplet lifetime $\tau_{T 1}^{T P c}=13 \mu \mathrm{s}$.

In this measurement, the maximum of the transient absorbance signal corresponding to the anthracene triplet excited state absorption $\Delta A_{e s a}^{a n t h}$ near 425 nanometers is $6.9 \mathrm{mOD}$. Following triplet transfer, the TPc bleach minimum near $650 \mathrm{~nm}$ is $-2.3 \mathrm{mOD}$ 
Therefore, anthracene triplet cross section in toluene is equal to (corrected for sub unity transfer efficiency and for between maximum instantaneous signal and total transfer efficiency):

$$
\begin{gathered}
\varepsilon_{T}^{a n t h}=\varepsilon_{g s b}^{T P c} * \frac{\Delta A_{e s a}^{a n t h}}{\Delta A_{g s b}^{T P c}} \frac{c_{T 1}^{T P c}}{c_{T 1}^{a n t h}}= \\
21000 * \frac{.0069}{.0023}\left(1-\frac{2}{2+25}\right)\left(1-\frac{2}{13+2}\right)=50,600 M^{-1} \mathrm{~cm}^{-1}
\end{gathered}
$$

\section{Determination of $\varepsilon_{T}^{P e p N o}$ for Polymer in Toluene}

A similar triplet sensitization procedure as for TPc was repeated for PePNo33, using similar concentrations of anthracene and polymer in solution. In the case, the triplet transfer time constant from anthracene to PePNo33 was determined to be $4.2 \mu$ s (25 $\mu$ s lifetime without PePNo33). Following sensitization, the lifetime of the PePNo33 triplet was determined to be $28.6 \mu \mathrm{s}$.

Using $\varepsilon_{T}^{\text {anth }}$ from the above analysis, we can directly determine $\varepsilon_{T}^{P e p N o}$ :

$$
\varepsilon_{T}^{P e P N o}=\varepsilon_{T 1}^{\text {anth }} * \frac{\Delta A_{e s a}^{P e P N o}}{\Delta A_{g s b}^{a n t h}} \frac{c_{T 1}^{\text {anth }}}{c_{T 1}^{P e P N o}}
$$

The polymer triplet signal has a maximum optical density during the sensitization measurement of $3.9 \mathrm{mOD}$ while the anthracene maximum absorption is $8.7 \mathrm{mOD}$. Therefore, the triplet cross section of the polymer in toluene, adjusting for decay of anthracene during transfer and decay of polymer triplet during transfer is:

$$
50,600 * \frac{3.9}{8.7} *\left(1 /\left(1-\frac{4.2}{4.2+25}\right)\right)\left(1 /\left(1-\frac{4.2}{4.2+28.6}\right)\right)=30,500 \mathrm{M}^{-1} \mathrm{~cm}^{-1}
$$

\section{Determination of Singlet Fission Yield}

Once all the molar extinction coefficients are known, the singlet fission yield is determined using a typical transient absorption data set, for direct excitation of dilute polymer in toluene. The 
maximum triplet absorption signal and corresponding ground state bleach are determined using global analysis procedures:

$$
\text { SF Yield }=\frac{\Delta A_{T}^{P e P N o}}{\Delta A_{S}^{P e P N o}} \times \frac{\varepsilon_{S}^{P e P N o}}{\varepsilon_{T}^{P e P N o}}=\frac{6.5 \mathrm{mOD}}{2.4 \mathrm{mOD}} \times \frac{23,615 \mathrm{M}^{-1} \mathrm{~cm}^{-1}}{30,500 \mathrm{M}^{-1} \mathrm{~cm}^{-1}}=209 \pm 20 \%
$$

\section{References}

(1) Sanders, S. N.; Kumarasamy, E.; Pun, A. B.; Trinh, M. T.; Choi, B.; Xia, J.; Taffet, E. J.; Low, J. Z.; Miller, J. R.; Roy, X.; Zhu, X. Y.; Steigerwald, M. L.; Sfeir, M. Y.; Campos, L. M. Quantitative Intramolecular Singlet Fission in Bipentacenes. J. Am. Chem. Soc. 2015, 137 (28), 8965-8972.

(2) Stern, H. L.; Musser, A. J.; Gelinas, S.; Parkinson, P.; Herz, L. M.; Bruzek, M. J.; Anthony, J.; Friend, R. H.; Walker, B. J. Identification of a Triplet Pair Intermediate in Singlet Exciton Fission in Solution. PNAS 2015, 112 (25), 7656-7661.

(3) Walker, B. J.; Musser, A. J.; Beljonne, D.; Friend, R. H. Singlet Exciton Fission in Solution. Nat. Chem. 2013, 5 (12), 1019-1024.

(4) Trinh, M. T.; Pinkard, A.; Pun, A. B.; Sanders, S. N.; Kumarasamy, E.; Sfeir, M. Y.; Campos, L. M.; Roy, X.; Zhu, X.-Y. Distinct Properties of the Triplet Pair State from Singlet Fission. Sci. Adv. 2017, 3 (7), e1700241.

(5) Tayebjee, M. J. Y.; Sanders, S. N.; Kumarasamy, E.; Campos, L. M.; Sfeir, M. Y.; McCamey, D. R. Quintet Multiexciton Dynamics in Singlet Fission. Nat. Phys. 2016, 13 (2), 182-188.

(6) Hore, M. J. A.; Hammouda, B.; Li, Y.; Cheng, H. Co-Nonsolvency of Poly( $n$ Isopropylacrylamide) in Deuterated Water/Ethanol Mixtures. Macromolecules 2013, 46 (19), 7894-7901.

(7) Lee, P. W.; Isarov, S. A.; Wallat, J. D.; Molugu, S. K.; Shukla, S.; Sun, J. E. P.; Zhang, J.; Zheng, Y.; Lucius Dougherty, M.; Konkolewicz, D.; Stewart, P. L.; Steinmetz, N. F.; Hore, M. J. A.; Pokorski, J. K. Polymer Structure and Conformation Alter the Antigenicity of Virus-like Particle-Polymer Conjugates. J. Am. Chem. Soc. 2017, 139 (9), 3312-3315.

(8) Hammouda, B.; IUCr. A New Guinier-Porod Model. J. Appl. Crystallogr. 2010, 43 (4), 716-719.

(9) Pesek, S. L.; Xiang, Q.; Hammouda, B.; Verduzco, R. Small-Angle Neutron Scattering Analysis of Bottlebrush Backbone and Side Chain Flexibility. J. Polym. Sci. Part B Polym. Phys. 2017, 55 (1), 104-111.

(10) Melanie S. Sanford; Jennifer A. Love, A.; Grubbs, R. H. A Versatile Precursor for the Synthesis of New Ruthenium Olefin Metathesis Catalysts. Organometallics 2001, 20 (25), 5314-5318.

(11) Plunkett, K. N.; Godula, K.; Nuckolls, C.; Tremblay, N.; Whalley, A. C.; Xiao, S. Expeditious Synthesis of Contorted Hexabenzocoronenes. Org. Lett. 2009, 11 (11), 22252228.

(12) Marie, A.; Fournier, F.; Tabet, J. C. Characterization of Synthetic Polymers by MALDITOF/MS: Investigation into New Methods of Sample Target Preparation and Consequence on Mass Spectrum Finger Print. Anal. Chem. 2000, 72 (20), 5106-5114. 